\title{
ECUACIONES PREDICTIVAS DEL MOVIMIENTO DEL SUELO PARA AMÉRICA CENTRAL, CON DATOS DE 1972 A 2010
}

\author{
GROUND MOTION PREDICTION MODELS FOR CENTRAL AMERICA USING \\ DATA FROM 1972 TO 2010
}

\section{Víctor Schmidt-Díaz}

\author{
Laboratorio de Ingeniería Sísmica, Instituto de Investigaciones en Ingeniería. \\ Nivel 3-A Facultad de Ingeniería, Universidad de Costa Rica. \\ 2060 San Pedro de Montes de Oca, San José, Costa Rica \\ victor.schmidt@ucr.ac.ce
}

(Recibido: 28/08/2013 ; aceptado: 5/03/2014)

\begin{abstract}
In the first part of the paper, the most important seismological aspects of Central America were reviewed. The predominant soil types and their origin were also studied. A description of the data used in aspects such as information sources, ranges of magnitude, hypocentral and epicentral distance, and earthquake classification according to their origin is presented. A procedure of soil classification for all sites is considered, based on the average spectral ratios obtained at each station, as well as in the geological maps and lithological descriptions. Attenuation equations are obtained for crustal earthquakes using data from Central America and for subduction earthquakes using records from Costa Rica. The functional form used is linear, taking the geometric mean of peak acceleration (GM PGA) or the geometric mean of the pseudo spectral acceleration (GM PSA) obtained from the response spectra for 5\% of damping, as the dependent variable. The moment magnitude, hypocentral distance, and soil type were used as independent variables. In the case of GM PSA, a total of 22 frequencies were considered. The results are compared with models proposed by other authors. In general it is observed that values obtained in this paper are lower. The response spectras were also compared with those proposed by the Seismic Code of Costa Rica. For all cases, the selected inversion method is the least-squares one step because it showed the highest stability of the results.

Keywords: Atttenuation equations, Central America, peak ground acceleration, spectral acceleration
\end{abstract}

RESUMEN: En la primera parte de esta publicación se realiza una revisión de los aspectos geológicos y sismológicos más importantes de cada zona identificando los principales tipos de suelo predominantes. Se realiza también una recopilación de datos acelerométricos de todos los países de América Central y se ha implementado un proceso de selección, homogeneización de formatos y clasificación de los datos teniendo en cuenta las fuentes de información, rangos de magnitudes, distancias consideradas y la forma en que se clasificaron los sismos según su origen. Se estableció además 
un procedimiento de clasificación de suelos para todos los sitios de registro, basado en las razones espectrales promedio obtenidas en cada estación, así como en la geología contenida en mapas y en descripciones litológicas. Se obtuvieron ecuaciones de atenuación para sismos corticales usando datos de América Central y para sismos por subducción usando registros de Costa Rica. El tipo de ecuación utilizado es lineal, considerando la media geométrica de la aceleración pico (GM PGA) o la media geométrica de la pseudo aceleración espectral (GM PSA), tomada del espectro de respuesta al $5 \%$ de amortiguamiento, en 22 frecuencias, como la variable dependiente y la magnitud momento, la distancia hipocentral y el tipo de suelo como las variables independientes. Los resultados se han comparado con modelos propuestos por otros autores y en general se observa que valores obtenidos en esta investigación son menores. Los espectros de respuesta fueron comparados también con los propuestos por el Código Sísmico de Costa Rica. Para realizar las inversiones, el método que ha mostrado mayor estabilidad en los resultados ha sido el de regresión de mínimos cuadrados de un paso.

Palabras clave: Ecuaciones de atenuación, América Central, aceleración pico, aceleración espectral

\section{INTRODUCCIÓN}

Uno de los usos más generalizados que se le da a nivel mundial a los registros de aceleración, lo constituye las relaciones de atenuación de la onda sísmica, ya que forman parte fundamental de los estudios de amenaza sísmica, tanto deterministas como probabilísticos. Autores como Rojas et al. (1998), han considerado que las ecuaciones de atenuación influyen en gran medida en los resultados que se obtengan en los estudios de amenaza. También conocidas como Ground Motion Prediction Equations (GMPE), estas ecuaciones son utilizadas además por ingenieros para estimar las fuerzas y/o desplazamientos a los que se pueden exponer las estructuras y con base en ellos, plantear las medidas correctivas requeridas para lograr un adecuado comportamiento estructural.

A nivel mundial, se han llevado a cabo una gran cantidad de estudios al respecto. Por ejemplo, Douglas (2004) hizo una recopilación de todas las ecuaciones de atenuación planteadas hasta esa fecha en el mundo y logró describir 165 modelos para PGA como variable dependiente y 100 ecuaciones obtenidas a partir de ordenadas espectrales.

La predicción empírica del movimiento del suelo parte de la relación existente entre una variable dependiente y de varias independientes, que representan las tres contribuciones principales al movimiento sísmico del suelo, que son: la fuente, la propagación y los efectos de sitio. Una expresión ampliamente aceptada para relacionar estos tipos de variables es la siguiente (Boore \& Joyner, 1982):

$$
\mathrm{Y}=\mathrm{b}_{1} \mathrm{e}^{\mathrm{b}_{2} \mathrm{M}}\left[\mathrm{e}^{\mathrm{b}_{3} \mathrm{D}} / \mathrm{D}^{\mathrm{b}_{4}}\right] \mathrm{e}^{\mathrm{b}_{5} \mathrm{~S}} \mathrm{e}^{\mathrm{b}_{6} \mathrm{P}}
$$

Aplicando logaritmos a ambos lados de la ecuación, queda en forma linealizada como:

$$
\ln \mathrm{Y}=\mathrm{C}_{0}+\mathrm{C}_{\mathrm{m}} \mathrm{M}-\mathrm{C}_{\mathrm{LD}} \ln \mathrm{D}-\mathrm{C}_{\mathrm{D}} \mathrm{D}+\mathrm{C}_{\mathrm{S}} \mathrm{S}+\mathrm{C}_{\mathrm{P}} \mathrm{P}
$$

Donde $\mathrm{Y}$ es la variable dependiente, que suele ser considerada como la aceleración pico del suelo, la aceleración espectral, la velocidad espectral o la velocidad pico del suelo. En esta investigación se trabajará con el primer caso.

Las variables independientes son $\mathrm{M}$ es la magnitud del sismo que generó el registro; en este caso será utilizada la $\mathrm{M}_{\mathrm{w}}$; D es una medida de distancia, que se considerará como la hipocentral (distancia más corta al hipocentro), debido a que es el dato disponible según las agencias consultadas. En este caso se considerará $D=\sqrt{ }\left(r^{2}+r_{h}^{2}\right)$, donde $r$ es la distancia hipocentral $\mathrm{y}_{\mathrm{h}}$ es un término ficticio introducido para resolver el problema de saturación del movimiento del suelo en el campo cercano (Dahle et al., 1995); S es una variable binaria que representa la geología local del sitio; $\mathrm{P}$ representa la incertidumbre en la predicción ( 0 
y 1 para los percentiles 50 y 84 , respectivamente) $\mathrm{y} \mathrm{C}_{0}, \mathrm{C}_{\mathrm{m}}, \mathrm{C}_{\mathrm{LD}}, \mathrm{C}_{\mathrm{D}}, \mathrm{C}_{\mathrm{S}} \mathrm{y} \mathrm{C}_{\mathrm{P}}$ son los parámetros a determinar en la regresión.

De acuerdo con Campbell (2003), generalmente las distancias epicentral y la hipocentral son malas medidas de D para ser consideradas en los modelos de atenuación para sismos grandes (magnitudes $\mathrm{M}_{\mathrm{w}}$ superiores a 6,5), debido a que estos sismos tienen áreas de ruptura extensas. Sin embargo, sí son distancias válidas principalmente para caracterizar sismos pequeños, que puedan ser razonablemente representados por una fuente puntual o cuando el plano de ruptura puede ser identificado por sismos ya sucedidos.

Sin embargo, en el caso de América Central existen muy pocos modelos de ruptura claramente definidos para sismos moderados y grandes, por lo que se debe optar por el uso de la distancia hipocentral.

De acuerdo con Boore \& Joyner (1982), es del criterio del investigador trabajar con parte o la totalidad de los términos de la ecuación 2, de acuerdo con la disponibilidad y fiabilidad de los datos, así como de las características propias de la región.

Los coeficientes $\mathrm{C}$ tienen el siguiente significado físico: $\mathrm{C}_{0}$ se refiere a la fuente generadora del sismo, $\mathrm{C}_{\mathrm{m}}$ es el coeficiente de escala de la magnitud, que también está relacionado con la fuente, $\mathrm{C}_{\mathrm{S}}$ con la condición de suelo y $\mathrm{C}_{\mathrm{P}}$ con la incertidumbre de la predicción.

Los términos $C_{L D}$ y $C_{D}$, que tienen que ver con la atenuación propiamente dicha, tanto elástica como inelástica requieren una especial descripción.

La expansión geométrica (elástica) está representada por el término $-\mathrm{C}_{\mathrm{LD}} * \ln \mathrm{n}$ en la ecuación 2 . Refleja la disminución de la amplitud de las ondas sísmicas en función de la distancia, debido a la variación de la energía por unidad de volumen, provocada por el aumento de la superficie del frente de ondas en un punto alejado de la fuente respecto otro más cercano. Cuando ocurre la ruptura, las ondas viajan en todas las direcciones; si la fuente es considerada como un punto y los frentes de onda como esféricos (válido en el campo cercano), puede ser demostrado que la amplitud de las ondas decrece con una razón $\mathrm{D}^{-1}$. Esta parte de la atenuación es causada por la naturaleza propia de la propagación de las ondas elásticas. El término
$-\mathrm{C}_{\mathrm{LD}} * \ln \mathrm{D}$ es descrito según el modelo propuesto por Herrmann \& Kijko (1983) como igual a G(D, $\mathrm{D}_{0}$ ), que resulta ser una propagación puramente esférica en el campo cercano (por debajo de una cierta distancia $\mathrm{D}_{0}$, comúnmente definida como $100 \mathrm{~km})$. En este caso, las amplitudes de las ondas decaen con la razón $\mathrm{D}^{-1}$ ya mencionada, siendo predominantes las ondas de volumen.

Sin embargo, esta expansión se vuelve cilíndrica para distancias mayores que $\mathrm{D}_{0}$, en la que predominan las ondas de superficie. En este caso, el decaimiento de las amplitudes de las ondas se da con una razón $\mathrm{D}^{-2}$.

En vista de la diferencia en cuanto al tipo de propagación con la distancia, la expresión $\mathrm{G}(\mathrm{D}$, $\mathrm{D}_{0}$ ) se simplifica a $C_{D}{ }^{*} \operatorname{Ln}(\mathrm{D})$, que resulta ser válida para todas las distancias y para la cuál se han observado los valores más bajos en la desviación estándar aunque se asuman otras expresiones (Climent et al., 1994).

La atenuación inelástica es representada por el término $-\mathrm{C}_{\mathrm{D}}{ }^{*} \mathrm{D}$ dentro de la ecuación $2 \mathrm{y}$ se basa en el hecho de que los materiales no son perfectamente elásticos; por lo tanto, si solo se tomara en cuenta la expansión geométrica dentro de la atenuación, el concepto quedaría incompleto. Físicamente, representa la pérdida de energía de las ondas sísmicas en forma de calor, como consecuencia de fricciones internas que presentan los materiales al viajar las ondas a través de ellos. Esta pérdida es debida a imperfecciones de la elasticidad de estos materiales.

El coeficiente $C_{D}$ se iguala a:

$$
\mathrm{C}_{\mathrm{D}}=\pi^{*} f / Q^{*} v
$$

Donde: $f$ es la frecuencia, $v$ es el promedio de las velocidades de onda cortante, $Q$ es el factor de calidad (adimensional), definido como $Q=Q_{\theta}{ }^{\text {n }}$ que mide la fricción interna o inelástica del material, si este se somete a ciclos de esfuerzos a una cierta frecuencia. $Q_{0}$ es una constante y $n$ es una constante positiva menor que la unidad. Valores altos de $Q$ reflejan materiales con comportamiento elástico, mientras $Q$ bajos se refieren a materiales menos elásticos, con mayor propensión a atenuar la energía (Tapia, 2006). 


\section{RESUMEN DE LA NEOTECTÓNICAY TIPOS DE SUELO DE AMÉRICA CENTRAL}

El istmo centroamericano se compone de 7 países: Guatemala, Belice, Honduras, El Salvador, Nicaragua, Costa Rica y Panamá, que cubren un área de aproximadamente $522760 \mathrm{~km}^{2}$, con $46 \mathrm{mi}-$ llones de personas en 2013, que corresponde a una densidad de población de 88 habitantes por $\mathrm{km}^{2}$.

Desde el punto de vista sismológico, la Fosa de América Central (o Fosa Mesoamericana) es la que representa la característica más notable de esta región. Se ubica en el océano Pacífico, desde la frontera de Guatemala y México en el norte hasta el sur de Costa Rica, donde se vuelve difusa. Esta fosa es el comienzo de la subducción de la placa del Coco (bloque de piso) debajo de la placa de Caribe (bloque de techo), por lo que se generan sismos de gran tamaño, incluso de magnitudes superiores a 8,0 , con mecanismos predominantemente inversos y la formación de numerosos volcanes activos en la parte continental (Montero et al., 1997).

La zona de subducción está dividida en varios segmentos de 100-300 km de longitud, cada uno con diferentes rumbos e inclinaciones y geometrías de la zona de Benioff, debido a las diferentes edades de la corteza oceánica. La subducción se inicia en la Fosa de América Central como se mencionó anteriormente, con fallas normalesdel lado de la ladera externa de la fosa, y fallas inversas a profundidades entre 15 y $50 \mathrm{~km}$ (Quintero \& Güendel, 2000; DeShon et al., 2003; Norabuena et al., 2004). A profundidades entre 50 y $280 \mathrm{~km}$, se producen sismos intraplaca o intra-slab (subducción profunda) y en general predominan mecanismos de tipo normal (Güendel \& Protti, 1998).

La zona de Benioff se vuelve menos profunda en el sur de Costa Rica, donde aparece la colisión de la Serranía del Coco. Hacia el este se ubica la Zona de Fractura de Panamá, que es un sistema de fallas dextral que separa la placa del Coco de la placa de Nazca.

Los terremotos más grandes de subducción se han producido en los segmentos de la fosa frente a las costas de Guatemala, El Salvador y Nicaragua.

El arco volcánico de Centroamérica, como otra consecuencia de la subducción, representa un área compleja y heterogénea. Se caracteriza por la ocurrencia de eventos sísmicos superficiales de magnitud baja a moderada (hasta 6.5), que históricamente han producido daños importantes, debido a que es uno de los arcos más densamente poblados del mundo. Las rupturas asociadas a esta zona sísmica son generalmente de desplazamiento lateral, de dirección longitudinal o transversal al arco (Montero et al., 1997).

Este arco se interrumpe abruptamente al sur de Costa Rica debido a la subducción de la litosfera oceánica más joven (Güendel \& Protti, 1998). Se introduce a la vez, en el marco del continente, la Serranía submarina del Coco, que llegó a la Fosa de América Central unos 2-3 Ma y que tiene un relieve topográfico elevado, generado por el punto caliente de Galápagos.

A la altura de Punta Burica (extremo sureste de Costa Rica) se forma un punto triple de interacción entre las placas del Coco, de Nazca y el bloque de Panamá. A lo largo de las costas del Pacífico panameño (istmo de Panamá), se ubica el límite de placa del Bloque de Panamá con la de Nazca, a pesar de no estar bien definido en su totalidad.

La placa del Caribe limita hacia el norte con el sistema de fallas Chixoy-Polochic, Motagua y Jocotán-Chamalecón, correspondiente al límite de placas entre las del Caribe y la de Norteamérica. Este límite está gobernado principalmente por movimiento transcurrente de componente lateral izquierdo (Güendel \& Protti, 1998).

En el Mar Caribe, frente a las costas de Costa Rica y Panamá, se encuentra el Cinturón Deformado de Panamá (CDNP), el cual es considerado como un sistema de fallas inversas y pliegues entre la placa del Caribe y el bloque de Panamá (Montero et al., 1997).

Por otro lado, la intensa actividad sísmica en Costa Rica entre 1990 y 1993 ha generado una teoría sobre la existencia de una zona de cizalla que atraviesa el país en un eje este-oeste. La zona sur de este bloque podría definir el norte del bloque de Panamá (Güendel \& Protti, 1998).

Este complejo marco tectónico de la región ha traído como consecuencia numerosos sismos destructivos: 52 con magnitudes entre 5,0 y 6,5 , 
ocurridos entre los años 1900 y 2002, principalmente generados en el arco volcánico y 51 con magnitudes mayores a 7,0, ocurridos fuera de dicha área, en las costas o cerca de ellas. (Rojas et al., 1993).

Los suelos predominantes en América Central son muy variados, como consecuencia de la complejidad del medio ambiente en el que interactúan diferentes tipos de roca, el clima, la topografía irregular y de los organismos vivos, incluidos los seres humanos.

En las montañas y valles a lo largo del eje central del istmo centroamericano predominan rocas volcánicas, incluyendo flujos de lava y diversas rocas piroclásticas, con excepción de un intrusivo situado en el sureste de Costa Rica. Las zonas de montaña se encuentran hacia la costa del Pacífico del istmo (sistemas montañosos generados por los procesos de subducción). Por otro lado, las llanuras costeras del Caribe dominan grandes extensiones. También, hay zonas aluviales en el lado pacífico del arco volcánico.

\section{RECOPILACIÓN, SELECCIÓN Y PROCESAMIENTO DE LOS DATOS}

Los acelerogramas utilizados para la obtención de las relaciones de atenuación deben provenir, en lo posible, de instrumentos digitales para evitar señales incompletas, saturadas o irregulares. Por esta razón, debido a que los acelerogramas más antiguos disponibles en la región son analógicos, se hace una selección visual (eliminando registros cortados) y calculada, considerando que la razón STA (short term average) respecto a LTA (long term average) sea entre 3 y 5 , tal y como lo proponen diversos autores para la diferenciación entre sismos y vibraciones ambientales (Atakan et al., 2004).

Una vez seleccionados los registros, se les aplican filtros pasa-banda, con rangos distintos para los analógicos y para los digitales, así como la corrección de la línea base. Se utiliza preliminarmente un filtro Ormbsby, con un rango de frecuencias de $0,25 \mathrm{~Hz}$ a $23 \mathrm{~Hz}$, para los registros que provienen de instrumentos analógicos y un rango de $0,12 \mathrm{~Hz}$ a $47 \mathrm{~Hz}$ para los digitales, tal y como lo recomiendan los fabricantes de los instrumentos, ya que prácticamente la totalidad de los registros fueron obtenidos con equipos de la marca Kinemetrics Inc, Pasadena, California. Para ambos tipos de instrumentos, el muestreo es de 200 muestras por segundo.

La figura 1 muestran la distribución de estaciones (a) y de epicentros (b) de todos los datos recopilados para este estudio. Sin embargo, tanto algunas estaciones como algunos registros fueron eliminados del análisis. Puede observarse que la mayoría de las estaciones preliminarmente seleccionadas se concentran en El Salvador y en Costa Rica, así como los epicentros de los sismos registrados.

Los datos de localización y profundidad de cada sismo fueron tomados del reporte de cada una de las agencias sismológicas de cada país, así como del CASC (Central American Seismological Center), dándole prioridad a los datos provenientes de agencias locales debido a que de ellos se espera mayor precisión, sobretodo en lo referente a sismos de magnitudes bajas y moderadas (inferiores a 5). Sin embargo, en muchos casos las diferencias entre los datos aportados por las instituciones consultadas fueron mínimas.

La información referente a las estaciones (coordenadas y geología local) fue reportada por cada agencia, así como el tipo de instrumento y número de serie asociado.

La separación entre eventos por subducción y corticales se llevó a cabo según la información reportada por cada agencia sismológica. Sin embargo, en la mayoría de los casos no existe un estudio específico al respecto, por lo que fue necesario asumir un criterio más simplificado basado en la profundidad (h), que corresponde al considerar eventos locales cuando $\mathrm{h}<25 \mathrm{~km}$ y de subducción cuando $h \geq 25 \mathrm{~km}$. Este criterio es sustentado de acuerdo a resultados obtenidos en estudios para Costa Rica (Sallares et al., 2000; DeShon et al., 2003; Warren, et al., 2008) o en observaciones obtenidas en Japón (Zhao et al., 2006a) que tiene un entorno sísmico similar a América Central.

Para la separación de eventos por subducción somera (interplaca) o profunda (intra-slab), se consideró que solamente los sismos clasificados 

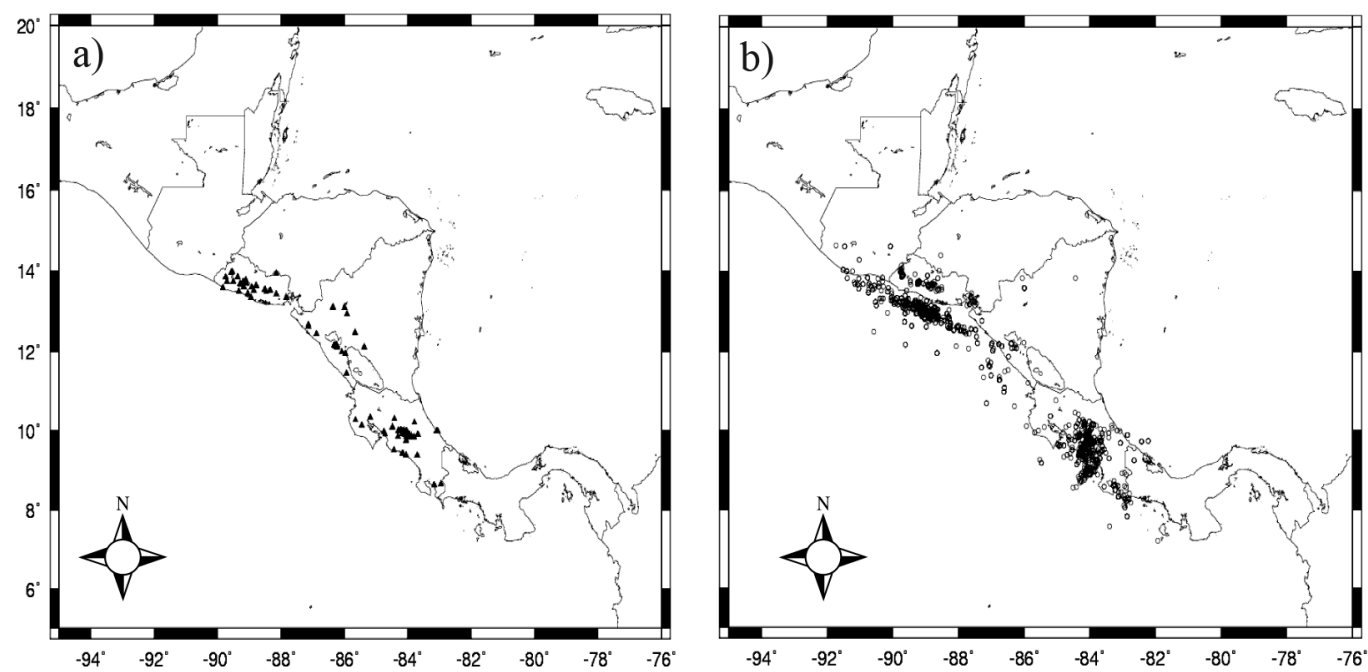

Fig. 1: Ubicación de las estaciones (a) y de los epicentros (b) de todos los datos recopilados para este estudio.

por las agencias como de subducción, pero con profundidades inferiores a $25 \mathrm{~km}$, corresponden a eventos de subducción somera o interplaca (J. Douglas, 2009, comunicación personal).

Finalmente, la magnitud utilizada es la $\mathrm{M}_{\mathrm{w}}$ (magnitud momento), que para la mayoría de los sismos con magnitud $\mathrm{M}_{\mathrm{w}} \geq 5$, fue directamente obtenida del Global Centroid Moment Tensor $C M T$. Si la magnitud era inferior o bien, no fue encontrada en la base de datos mencionada, fue necesario el uso de correlaciones.

Tomando en cuenta el hecho de que muchos eventos reportados por agencias locales tienen solamente asignada la magnitud $\mathrm{M}_{\mathrm{D}}$ (local o de duración), fue necesario el uso de las siguientes regresiones propuestas por varios autores (Okal \& Romanovicz, 1994):

$$
\mathrm{M}_{\mathrm{w}}=2,27+2 / 3 * \mathrm{M}_{\mathrm{s}}
$$

Donde $\mathrm{M}_{\mathrm{s}}$ es la magnitud obtenida a partir de ondas superficiales (Rojas et al., 1993):

$$
M_{s}=-4,165+1,783 * M_{D}
$$

Y combinando estas dos ecuaciones se obtiene:

$$
\mathrm{M}_{\mathrm{w}}=-0,507+1,186 * \mathrm{M}_{\mathrm{D}}
$$

De esta forma, es posible unificar las magnitudes $M_{w}$ ya que es un requisito básico para plantear las relaciones de atenuación.

Otro de los términos más importantes en las ecuaciones predictivas de movimiento fuerte, es el que corresponde a la respuesta del suelo, conocido también como efectos de sitio. Para esto, resulta vital contar con una clasificación confiable de los tipos de suelo, donde se ubican las estaciones acelerográficas que se tomarán en cuenta en el análisis, lo que permitirá obtener modelos más robustos y confiables.

En América Central las redes de cada país cuentan con una clasificación de suelos propia (Bundschuh \& Alvarado, 2007), pero esta resulta ser subjetiva y difícilmente comparable, ya que se basa en interpretaciones de mapas geológicos y geotécnicos, o bien, en pruebas que se realizaron sin seguir un procedimiento estándar.

En estudios realizados en diversas partes del mundo, se han observado grandes variaciones en los resultados de estudios de peligrosidad sísmica, obtenidos con base en ecuaciones que predicen el movimiento fuerte del suelo, a partir de registros en sitios cuya clasificación de suelo es poco confiable.

Por estas razones, se aplicó el método de clasificación propuesto por Zhao et al. (2006b) basado en el cálculo de razones espectrales para sismos y 
cuyos resultados para Costa Rica se pueden encontrar en forma amplia en Schmidt (2011a).

Este consiste en términos muy generales, en la obtención de la razón espectral $\mathrm{H} / \mathrm{V}$ promedio para cada estación obtenida a partir de todos los sismos registrados en esa estación y con base en ella, se calcula un índice de clasificación, que permite determinar si este es S I (roca), S II (suelo firme), S III (suelo medio) y S IV (suelo blando). En general, existen pocas estaciones bien calificadas correspondientes a S I y también pocas del tipo S IV.

El hecho de que pocas estaciones hayan sido clasificadas como suelo tipo roca es concordante con el tipo de geología predominante en los tres países, sobretodo en las zonas donde se ubican la mayor cantidad de los instrumentos (valles, zonas costeras, zonas inundables).

Fue encontrado un porcentaje similar de estaciones satisfactoriamente clasificadas para Costa Rica y Nicaragua (aproximadamente un 64\% en ambos casos), e inferior para El Salvador (un 52\%).

Para efectos de simplificación y en vista de que no existe una gran cantidad de registros correspondientes a suelo S IV, se trabajó con tres categorías: S I que equivale a roca, S II a suelo firme y (S III + S IV) a suelo blando.

Respecto a la variable dependiente, en vista de que existen dos componentes horizontales por registro, se calculó la media geométrica, definida como:

$$
\mathrm{GM}(\mathrm{Y})=\sqrt{ }\left(\mathrm{Y}_{\mathrm{X}} * \mathrm{Y}_{\mathrm{Y}}\right)
$$

Donde:

Y es la ordenada que se usará en el estudio de regresión, que puede ser PGA o PSA.

$\mathrm{Y}_{\mathrm{x}}$ es la ordenada en la componente N-S.

$\mathrm{Y}_{\mathrm{y}}$ es la ordenada en la componente E-O.

Para el análisis, los sismos se limitaron a magnitudes $M_{w} \geq 4.0$, para asegurar la validez de los modelos para magnitudes entre 5 y 7 .

De forma semejante, las distancias hipocentrales utilizadas son las inferiores a $200 \mathrm{~km}$, ya que valores mayores no resultan ser importantes para estudios en el ámbito de la ingeniería ( $\mathrm{J}$. Douglas 2009, comunicación personal).
Los modelos que se proponen se obtuvieron con base en el programa de libre distribución, creado por Douglas, J. (2009, comunicación personal), última versión, en MathLab $\mathbb{C}$, que permite seleccionar entre 15 tipos de ecuaciones o formas de las funciones y cinco métodos de regresión, entre ellos: regresión ordinaria de un paso y dos pasos (mínimos cuadrados) y máxima verosimilitud de un paso y de dos pasos. En el siguiente apartado se hace referencia al modelo que mejor se ajusta a cada regresión.

\section{RESULTADOS}

\section{Ecuaciones para GM PGA para eventos corticales}

Se obtienen las ecuaciones de atenuación para sismos corticales de América Central, también llamados de fallamiento local, para la media geométrica de la aceleración pico (GM PGA) y para la media geométrica de la pseudo aceleración (GM PSA) correspondiente a 22 frecuencias.

El cuadro 1 muestra un resumen de los datos usados. Corresponde a 439 registros de 212 eventos, la mayor parte de ellos registrados en suelo S II (firme) y solamente un $10 \%$ en S I (roca). Esto era de esperar ya que como se mencionó, son pocos los sitios de roca donde se ubican estaciones acelerográficas en América Central, debido a que no es una condición de suelo común de acuerdo con la geología predominante en la región.

Según los rangos de magnitudes, la mayoría de los registros (un 58\%) corresponden a sismos con $\mathrm{M}_{\mathrm{w}}$ entre 4 y 4,9 y solamente un $14 \%$ a sismos $\operatorname{con} \mathrm{M}_{\mathrm{w}} \geq 6,0$.

La figura 2a muestra la distribución de las magnitudes respecto a la distancia hipocentral. Es notoria la ausencia de datos para magnitudes superiores a 6,5 , correspondientes a distancias entre 10 y $90 \mathrm{~km}$, separación mostrada en la misma figura por la línea inclinada. Para el resto de distancias y magnitudes, la cobertura de los datos es densa y están casi uniformemente repartidos. 
Cuadro 1

Resumen de los datos usados para la obtención de las relaciones de atenuación considerando sismos de fallamiento cortical.

\begin{tabular}{cccc}
\hline Número de eventos & Número de registros & Según tipo de suelo & Según rango de magnitudes \\
\hline 212 & 439 & S I: $43(10 \%)$ & 4 a 4,9: $256(58 \%)$ \\
& S II: 246 (56\%) & 5 a 5,9: $12028 \%)$ \\
& S III y S IV: $151(34 \%)$ & 6 o más: $63(14 \%)$ \\
\hline
\end{tabular}

Respecto a la distribución de los datos según el tipo de suelo, la figura 2 b muestra la poca presencia de datos correspondientes a roca en general, así como pocos puntos para suelo firme y blando para magnitudes superiores a 6 , entre distancias de 10 a $90 \mathrm{~km}$. Se observa también más densidad de puntos correspondientes a suelo firme entre magnitudes de 4 a 5 , situación que disminuye para magnitudes mayores.

Respecto a la distribución por magnitudes, la figura 3a muestra claramente la diferenciación entre los distintos rangos, estando los valores más bajos asociados a magnitudes entre 4 y 4,9, los intermedios entre 5 y 5,9 y los más altos con $\mathrm{M}_{\mathrm{w}}$ superiores a 6 .

La figura $3 \mathrm{~b}$ presenta la distribución de la media geométrica del PGA, en adelante GM PGA, en función de la distancia hipocentral para



los distintos tipos de suelo. Se nota la poca presencia de datos para roca y no se diferencian claramente las distribuciones para las tres condiciones de suelo consideradas, ya que todos se distribuyen más o menos en forma homogénea en la nube de puntos. Se observa sutilmente una tendencia a la disminución de GM PGA con respecto al aumento de la distancia hipocentral, como era de esperarse, lo que se muestra por medio de una línea diagonal dibujada sobre el mismo gráfico.

Después de realizar una serie de pruebas respecto a estabilidad de los modelos y valores de desviaciones estandar, la ecuación seleccionada tiene la siguiente forma:

$$
+\mathrm{H}^{*} \mathrm{C}_{6}
$$$$
\log \mathrm{Y}=\mathrm{C}_{1}+\mathrm{C}_{2} * \mathrm{M}+\mathrm{C}_{3} * \log \left(\operatorname{sqrt}\left(\mathrm{D}^{2}+\mathrm{C}_{4}^{2}\right)\right)+\mathrm{S} * \mathrm{C}_{5}
$$



Fig. 2: a) Gráfico de $M_{w}$ vs. distancia hipocentral para todos los sismos clasificados como originados por corteza superficial. b) Gráfico de $\mathrm{M}_{\mathrm{w}}$ vs. distancia hipocentral con separación por tipo de suelo. 



Fig. 3: a) GM PGA vs. distancia hipocentral para distintos rangos de magnitudes. b) GM PGA vs. distancia hipocentral según los distintos tipos de suelo.

Donde el término $\mathrm{C}_{\mathrm{s}} \mathrm{S}$ de la ecuación se ha divido en $\mathrm{S} * \mathrm{C}_{5}+\mathrm{H}^{*} \mathrm{C}_{6}$ y el coeficiente $\mathrm{C}_{\mathrm{p}}$ es igual a cero por tratarse del percentil 50. Y es la GM PGA o GM PSA en $\mathrm{cm} / \mathrm{s}^{2}, \log$ corresponde al logaritmo base $10, \mathrm{C}_{1}$ a $\mathrm{C}_{6}$ son los términos a ser obtenidos por medio de la regresión y no es considerado el término correspondiente a la atenuación inelástica, justificado además porque este tiene un efecto importante en las distancias lejanas (superiores a los $200 \mathrm{~km}$ ) y este estudio se limitó a D inferior a 200 km (Schmidt, 2010). Los dos últimos términos corresponden a la condición del suelo en el sitio de registro; en caso de que sea roca (S I), S $=\mathrm{H}=0$, si el suelo es firme (S II), $\mathrm{S}=0 \mathrm{y} \mathrm{H}=1$ y para suelo blando (S III y $\mathrm{S} \mathrm{IV}$ ) $\mathrm{S}=1$ y $\mathrm{H}=0$. Los métodos de regresión que mostraron mayor estabilidad corresponden a la ordinaria de un paso (mínimos cuadrados) y no a los de una regresión ordinaria de dos pasos (Joyner \& Boore, 1981), ni al método de máxima verosimilitud (Joyner \&
Boore, 1993), ya que estos dos últimos procedimientos mostraron grandes inestabilidades, sobretodo para PSA en las frecuencias bajas.

Los coeficientes que mejor ajustaron la ecuación se muestran en el cuadro 2. El término referente a la atenuación elástica $\left(\mathrm{C}_{3}\right)$ adquiere un valor cercano a -1,0, lo que parece indicar que se está en presencia de una propagación de ondas tipo esférica. Los que determinan la amplificación por presencia de suelo firme y blando respecto a la roca, tienen un valor de $1,66\left(10^{0,22}\right)$ para suelo blando y $1,32\left(10^{0,12}\right)$ para suelo firme, lo que significa que estos tipos de suelo amplifican respecto a la roca un $66 \%$ y un $32 \%$ respectivamente, para GM PGA.

Las figuras $4 a, 4 b, 4 c$ y $4 d$ muestran los resultados obtenidos para las tres condiciones de suelo: $\mathrm{S}$ I, S II y S III para $\mathrm{M}_{\mathrm{w}}=6$ y se muestran los valores observados (puntos negros) transformados al aplicar los coeficientes de suelo resultantes a las observaciones en suelos distintos al caso considerado. Se

Cuadro 2

Coeficientes obtenidos de acuerdo con el modelo de inversión más estable para GM PGA, sismos corticales.

\begin{tabular}{ccccccc}
\hline $\mathrm{C} 1$ & $\mathrm{C} 2$ & $\mathrm{C} 3$ & $\mathrm{C} 4$ & $\mathrm{C} 5$ & $\mathrm{C} 6$ & $\mathrm{SD}$ \\
\hline 0,12602 & 0,49081 & $-1,03591$ & 4,22442 & 0,22075 & 0,11742 & 0,4078 \\
\hline
\end{tabular}





Fig. 4: Resultados obtenidos para roca (a), suelo firme (b), suelo blando (c) y los tres tipos de suelo (d), para GM PGA y magnitud $\mathrm{M}_{\mathrm{w}}=6$. En las figuras a, b y c se muestran además las desviaciones estándar (SD) y las observaciones (triánngulos) a partir de las cuales se obtuvieron las regresiones, que fueron transformadas si la observación corresponde a un caso distinto (suelo diferente) al considerado.

muestran además las curvas correspondientes a la suma y a la resta de una desviación estándar $(+1$ SD y -1 SD). Se observa un adecuado ajuste visual de los datos dentro de los rangos mencionados, aún cuando la desviación estándar pueda ser considerada alta de acuerdo con otros autores (Douglas, 2009; Bungum, 2009, comunicación personal).
Los residuos representados en la figura 5 se obtuvieron de acuerdo con la relación:

$$
\text { residuo }(y)=\log \left(o b s_{y}\right)-\log \left(\text { pred }_{y}\right)
$$

Donde: $y$ corresponde a cada entrada (registro), $o b s_{y}$ al valor observado, que en este caso es 
la GM PGA obtenida del acelerograma y pred al valor obtenido con base en la ecuación y con el uso de los coeficientes del cuadro 2 .

De acuerdo con esta ecuación, el valor ideal se alcanza cuando el residuo $(y)=0$. Sin embargo, es de esperarse que los valores que predicen las ecuaciones sean mayores o menores que las observaciones, tomando en cuenta la forma en que se distribuyen los datos con la distancia y la magnitud. De esta forma, se obtienen tres residuos en un rango entre 1,5 y 2,5 (observación mayor que el valor generado por el modelo) y seis entre -1,5 y -2,0 (caso inverso). La mayoría de residuos se ubican entre +1 y -1 , que suele ser lo común de acuerdo con resultados obtenidos por diversos autores.

En la figura 5a, se grafican los residuos en función de la distancia hipocentral, observándose una clara tendencia al decrecimiento, que puede ser representada por una ecuación lineal cuyo residuo adquiere un valor igual a cero a los $45 \mathrm{~km}$. Cuando se grafican en función de la magnitud $\mathrm{M}_{\mathrm{w}}$, se observa también un sesgo al decrecimiento al aumentar la magnitud, representado por una línea recta, pero con una pendiente menor que la anterior (Fig. 5b). En este caso, los residuos adquieren un valor igual a cero para una magnitud $\mathrm{M}_{\mathrm{w}}=5,0$.

Finalmente, la figura 6 muestra una comparación entre los resultados obtenidos en este estudio con respecto a otros autores. Se observa que en



el campo cercano (hasta los $10 \mathrm{~km}$ de distancia hipocentral), la ecuación propuesta es cercana al promedio de las demás mostradas. Sin embargo, para valores mayores los otros modelos superan el obtenido con datos de América Central, sobretodo porque este tiene una caída prácticamente lineal entre los 10 y $200 \mathrm{~km}$. Esto se puede deber en gran medida a los métodos de regresión empleados, ya que en este estudio el método de mínimos cuadrados, es el que mejor se adaptó a los datos disponibles y otros autores han utilizado el de máxima verosimilitud (Zhao et al., 2006a; Spudich et al., 1999), así como el método de Bayes (Dahle et al., 1995; Schmidt et al., 1997). Si se usa el de Bayes, al utilizar conocimiento científico previo, el valor de cada coeficiente se restringe a rangos previamente estudiados y se logra por ejemplo, que los coeficientes correspondientes a suelo firme o blando siempre amplifiquen respecto a la roca.

Especial atención merece la comparación con los modelos propuestos por Dahle et al. (1995), ya que también se basan en datos que provienen de América Central. Los resultados muestran diferencias prácticamente en todo el rango de distancias. Sin embargo, debe aclararse que para el presente estudio fueron eliminados prácticamente todos los registros provenientes de instrumentos analógicos, que fueron los predominantes en el estudio de 1995. Además, como se dijo los métodos de inversión se basan en principios distintos, por lo que



Fig. 5: Representación de los residuos en función de la distancia hipocentral (a) y de la $\mathrm{M}_{\mathrm{w}}$ (b), para GM PGA correspondientes a registros de fallamiento cortical de América Central. 
— Este estudio — Schmidt et al, 1997

—Dahle 1995 — Zhao 2006 — Spudich 1999



Distancia hipocentral $(\mathrm{km})$

Fig. 6: Comparación de las relaciones de atenuación obtenidas en este estudio con las propuestas por otros autores, para datos de fallamiento cortical, $\mathrm{M}_{\mathrm{w}}=6 \mathrm{y}$ en condición de roca.

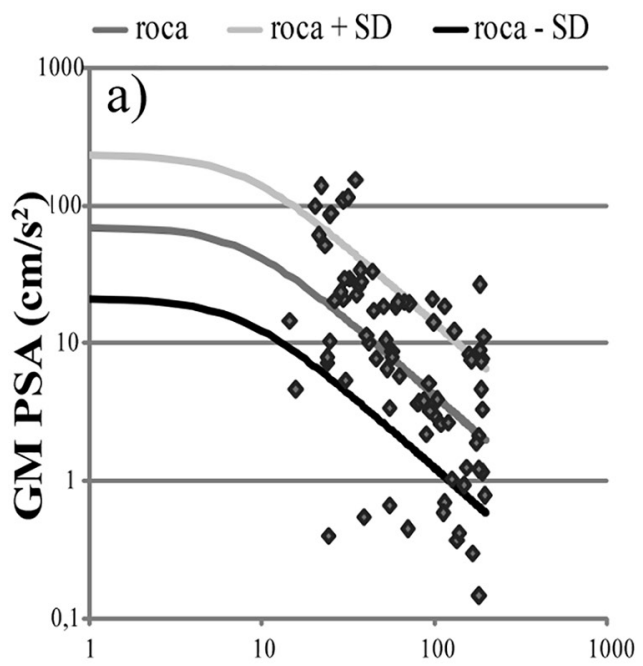

Distancia hipocentral $(\mathrm{km})$ los resultados no son directamente comparables, a pesar de que los datos provengan de un mismo entorno sísmico.

\section{Ecuaciones para GM PSA para eventos corticales}

En este apartado se presentan los resultados obtenidos para la media geométrica de la pseudo aceleración (GM PSA) para 22 periodos seleccionados.

Respecto a la GM PSA $1 \mathrm{~Hz}$, se observa en la figura 7a para roca y $\mathrm{M}_{\mathrm{w}}=6$ con su respectiva desviación estándar y en la figura $7 \mathrm{~b}$ se muestran las tres curvas que representan los tres tipos de suelo considerados. En este caso, es más notoria la diferenciación entre suelo S III (blando) respecto a S I (roca), ya que S II y S I aparecen como curvas más cercanas. Esta situación es más evidente que en el caso de GM PGA, donde las tres curvas son más equidistantes (Fig. 4d), situación esperable, ya que para $1 \mathrm{~Hz}$ PSA, la amplificación por presencia de suelos blandos suele ser mayor que para frecuencias altas, representadas por el PGA, que se asocia con frecuencias mayores que $40 \mathrm{~Hz}$.



Distancia hipocentral (km)

Fig. 7: Relaciones de atenuación obtenidas para GM PSA $1 \mathrm{~Hz}$, para $\mathrm{M}_{\mathrm{w}}=6$. Se muestran los resultados para condición de roca, con la desviación estándar (líneas punteadas) y las observaciones (puntos negros (a) y las relaciones para las tres condiciones de suelo (b). 
Considerando los residuos, no se observa un sesgo o tendencia en su distribución respecto a la distancia hipocentral o a la magnitud, ya que la línea de mejor ajuste coincide con la línea de residuo igual a cero. Se obtienen además residuos máximos alrededor de 1,5 y -2 , que son menores que para el caso de GM PGA (figuras 8a y $8 b$ ).

La figura 9 compara las ecuaciones obtenidas por distintos autores para GM PSA respecto a la distancia hipocentral. Es evidente que el modelo propuesto en este estudio se mantiene por debajo de los demás en todo el rango de distancias. En varios de los modelos propuestos por otros autores, es utilizada la distancia a la zona de ruptura o la propuesta por Joyner \& Boore (1981) y en el presente estudio es la distancia hipocentral, que como ya se dijo es mayor, por lo que este factor puede justificar en parte los resultados observados.

El cuadro 3 muestra los resultados de los coeficientes obtenidos de GM PSA como variable dependiente para 22 periodos, así como la desviación estándar y las Figuras 10a, 10b, 10c, 10d, 10 e y $10 \mathrm{f}$ muestran la variación de los coeficientes en función del periodo.

En la figura 10a se observa que $\mathrm{C} 1$ aumenta ligeramente hasta llegar a un máximo en 0,1 s y luego disminuye. El rango de variación de este coeficiente va de 0,75 a $-3,5$. Para este coeficiente, que define la forma general del espectro

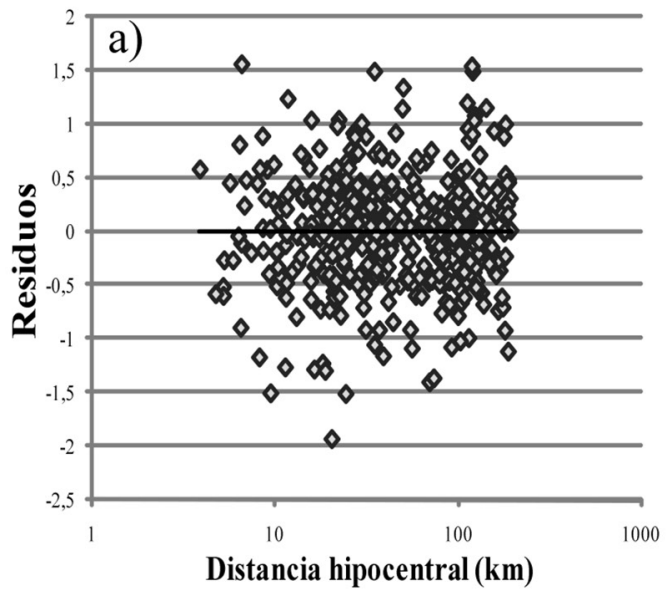

de respuesta, ya que es un coeficiente de escala, se observa un comportamiento de acuerdo con lo esperado, observándose un máximo en $0,15 \mathrm{~s}$.

En la figura 10b, se observa un aumento de $\mathrm{C}_{2}$ (coeficiente que escala la magnitud) de 0,45 a 0,9 en un rango de periodos de $0,02 \mathrm{~s}$ a $2 \mathrm{~s}$. Esto muestra que la magnitud tiene una influencia mayor en periodos largos a partir de $0,9 \mathrm{~s}$, lo cual es también un comportamiento esperado y observado por autores como García et al. (2005) y Atkinson \& Boore (2003).

En la figura 10c, se observa que no existe una tendencia definida para la variación de $\mathrm{C}_{3}$ (expansión geométrica) en función del periodo, obteniéndose un máximo en $0,4 \mathrm{~s}$ de $-0,99$ y un mínimo en $0,15 \mathrm{~s}$ de $-1,14$, siendo este rango de variación pequeño, por lo que puede ser considerado un coeficiente bastante estable. En este caso, todos los valores obtenidos están en torno a $-1,0$, por lo que se asume una propagación de ondas esférica (Tapia, 2006).

La variación de $\mathrm{C}_{4}$ (distancia ficticia introducida) no sigue una tendencia particular (Fig. 10d), mostrando un rango de valores de 2,15 a 9,4.

En la figura 10f se muestra la variación de la desviación estándar, donde se observa un valor estable entre $0,02 \mathrm{~s}$ y $0,15 \mathrm{~s}$, cercano a 0,4 , un incremento entre 0,15 s y 0,9 s de 0,4 a 0,5 y nuevamente se estabiliza a partir de $0,9 \mathrm{~s}$ en 0,5 . Este



Fig. 8: Residuos para GM PSA 1 en función de la distancia hipocentral (a) y en función de la magnitud (b). Ambos casos para sismos por fallamiento cortical. 
—Este estudio —Schmidt et al, 1997

—Dahle 1995 —Zhao 2006 —Spudich 1999



Distancia hipocentral $(\mathrm{km})$

Fig. 9: Comparación de la relación de atenuación obtenida en este estudio (línea más oscura) con otros modelos propuestos por diversos autores, para GM PSA $1 \mathrm{~Hz}, \mathrm{M}_{\mathrm{w}}=6,0$, roca, PSA para $1 \mathrm{~Hz}$. .

incremento en función del periodo puede estar asociado a los procesos de registro y procesado de la señal, ya que aún cuando se han aplicado los filtros señalados, los periodos largos pueden estar más influenciados por estos procesos.

Respecto a los coeficientes que representan la amplificación del suelo $\left(\mathrm{C}_{5} \mathrm{y}_{6}\right)$, la figura $10 \mathrm{e}$ muestra la variación de ambos en función del periodo. Estos se mantienen más o menos estables y semejantes entre $0,02 \mathrm{~s}$ y $0,15 \mathrm{~s}$, pero a partir de allí el coeficiente b5 correspondiente a suelo blando sufre un gran incremento hasta llegar a un valor de 3,66 $\left(10^{\mathrm{b} 5}\right)$ en $0,44 \mathrm{~s}$. El coeficiente $\mathrm{C}_{6}$ correspondiente a suelo firme sufre también un incremento pero mucho menor, llegando a un máximo de $1,9\left(10^{\mathrm{b} 6}\right)$ en $0,44 \mathrm{~s}$. Sin embargo, a partir de este periodo existe un factor de 2 entre $\mathrm{C}_{5} \mathrm{y} \mathrm{C}_{6}$. Otros análisis hechos a partir de los coeficientes de suelo estimados pueden encontrarse en Schmidt (2011b).

La figura 11 compara los espectros obtenidos en este estudio para $\mathrm{M}_{\mathrm{w}}=6$ y la distancia hipocentral de $50 \mathrm{~km}$ para dos condiciones de suelo: S II y S III. Debido a que en casos como Dahle et al. (1995) y Schmidt et al. (1997) se reportan valores de PGA y PSA máximos, se siguió el procedimiento propuesto por Beyer \& Bommer (2006) para transformar esos valores a GM PGA y GM PSA, respectivamente. No se compara el caso de $\mathrm{S}$ I debido a la poca cantidad de datos para esa clasificación del sitio, según los datos disponibles para la zona en estudio.

Para la condición S II, los espectros obtenidos en este estudio son inferiores a los propuestos por otros autores (Fig. 11a). No se comparó con Dahle et al. (1995) debido a que esta condición de suelo no fue considerada explícitamente en ese estudio.

La figura $11 \mathrm{~b}$ muestra la comparación de los espectros para suelo blando (S III) y se observa una semejanza considerable entre las ordenadas espectrales desde 0,1 a $10 \mathrm{~s}$ de periodo. Esta semejanza de GM PSA está asociada a los altos factores de amplificación obtenidos en el presente estudio para la condición de suelo S III en ese rango de periodos.

Finalmente, en la figura 12 puede apreciarse la comparación de los espectros obtenidos a partir de las ecuaciones de atenuación planteadas para sismos de origen cortical con los espectros de diseño propuestos por el Código Sísmico de Costa Rica (CFIA, 2002), para 500 años de periodo de retorno, cuyas ordenadas espectrales fueron transformadas de aceleración pico efectiva a PGA según Laporte (2006).

Se observa que el espectro propuesto por la norma citada cubre todas las ordenadas espectrales obtenidas para un sismo de origen cortical de magnitud $\mathrm{M}_{\mathrm{w}}=7 \mathrm{y}$ una distancia hipocentral (D) de $10 \mathrm{~km}$, para emplazamientos de roca en San José, Costa Rica, por lo que puede afirmarse que sismos de magnitudes y distancias hipocentrales inferiores también estarán cubiertos por dicha norma.

Por medio de la figura $12 \mathrm{~b}$ se compara el espectro propuesto para Costa Rica por la norma para condición de suelo firme en San José, con el correspondiente a un sismo cortical de las mismas características que el anterior $\left(\mathrm{M}_{\mathrm{w}}\right.$ $=7 \mathrm{y} \mathrm{D}=10 \mathrm{~km}$ ). En este caso se observa una 



Fig. 10: Variación de los coeficientes c1, c2, c3 y c4 y de la desviación estándar SD según el Cuadro 3 en función del periodo, para sismos corticales con datos de América Central.

gran similitud entre ambos hasta un periodo de $1 \mathrm{~s}$, a partir del cual la norma se vuelve conservadora debido a que el decaimiento observado en el espectro obtenido con las ecuaciones propuestas es más fuerte.
La figura $12 \mathrm{~b}$ compara el espectro de la norma de Costa Rica para condición de suelo blando con dos espectros obtenidos con las ecuaciones de atenuación propuestas, que nuevamente muestran un decaimiento más fuerte respecto al espectro 
Cuadro 3

Variación de los coeficientes C1 a C6 y de la desviación estándar para 22 periodos considerados, correspondientes a eventos corticales.

\begin{tabular}{|c|c|c|c|c|c|c|c|c|}
\hline Period & frec & $\mathrm{C} 1$ & $\mathrm{C} 2$ & $\mathrm{C} 3$ & $\mathrm{C} 4$ & $\mathrm{C} 5$ & C6 & SD \\
\hline 0,02 & 50,00 & 0,15454 & 0,48743 & $-1,03269$ & 3,83891 & 0,21489 & 0,11115 & 0,4093 \\
\hline 0,075 & 13,33 & 0,65109 & 0,44289 & $-1,06921$ & 2,15714 & 0,21033 & 0,16857 & 0,4131 \\
\hline 0,1 & 10,00 & 0,73993 & 0,45503 & $-1,08638$ & 4,65588 & 0,15027 & 0,12146 & 0,4105 \\
\hline 0,15 & 6,67 & 0,75961 & 0,48369 & $-1,14082$ & 8,92792 & 0,17034 & 0,07779 & 0,4194 \\
\hline 0,2 & 5,00 & 0,47439 & 0,51668 & $-1,12103$ & 8,40521 & 0,24001 & 0,1382 & 0,4394 \\
\hline 0,24 & 4,17 & 0,13594 & 0,53838 & $-1,06081$ & 6,43782 & 0,35122 & 0,18941 & 0,4533 \\
\hline 0,303 & 3,30 & $-0,30862$ & 0,57998 & $-1,02221$ & 4,81306 & 0,49143 & 0,25633 & 0,4727 \\
\hline 0,34 & 2,94 & $-0,5482$ & 0,60292 & $-0,99302$ & 3,71378 & 0,53045 & 0,26343 & 0,485 \\
\hline 0,4 & 2,50 & $-0,83868$ & 0,64157 & $-0,99596$ & 3,50109 & 0,55494 & 0,27397 & 0,4936 \\
\hline 0,44 & 2,27 & $-1,00207$ & 0,66931 & $-1,01606$ & 4,30379 & 0,56379 & 0,26458 & 0,4934 \\
\hline 0,5 & 2,00 & $-1,20878$ & 0,70917 & $-1,05064$ & 5,31951 & 0,55509 & 0,24432 & 0,4937 \\
\hline 0,6 & 1,67 & $-1,43766$ & 0,74588 & $-1,07315$ & 6,41843 & 0,52324 & 0,18262 & 0,5041 \\
\hline 0,752 & 1,33 & $-1,82261$ & 0,80402 & $-1,11353$ & 7,88318 & 0,49587 & 0,17859 & 0,5153 \\
\hline 0,9 & 1,11 & $-2,14054$ & 0,84448 & $-1,11862$ & 7,77669 & 0,45466 & 0,14805 & 0,5293 \\
\hline 1 & 1,00 & $-2,26639$ & 0,85062 & $-1,10729$ & 7,93455 & 0,43811 & 0,13807 & 0,5303 \\
\hline 1,25 & 0,80 & $-2,46703$ & 0,85888 & $-1,1121$ & 9,40068 & 0,418 & 0,1332 & 0,521 \\
\hline 1,493 & 0,67 & $-2,76269$ & 0,87686 & $-1,09131$ & 7,9465 & 0,409 & 0,12784 & 0,5245 \\
\hline 2 & 0,50 & $-3,1279$ & 0,9095 & $-1,11692$ & 7,67692 & 0,39708 & 0,10211 & 0,5324 \\
\hline 2,5 & 0,40 & $-3,24945$ & 0,89781 & $-1,11774$ & 7,38462 & 0,36476 & 0,09728 & 0,527 \\
\hline 3,03 & 0,33 & $-3,33051$ & 0,87247 & $-1,09664$ & 6,02374 & 0,3585 & 0,09856 & 0,5288 \\
\hline 4 & 0,25 & $-3,40089$ & 0,83189 & $-1,06428$ & 3,28831 & 0,33733 & 0,08206 & 0,5277 \\
\hline 5 & 0,20 & $-3,41634$ & 0,80107 & $-1,06854$ & 2,46111 & 0,33726 & 0,08534 & 0,5403 \\
\hline
\end{tabular}

del código. Se puede concluir entonces que para este caso, el espectro propuesto por la norma se ubica entre dos posibles sismos: uno de $\mathrm{M}_{\mathrm{w}}=7,0$ y $\mathrm{D}=10 \mathrm{~km}$ por encima y otro de $\mathrm{M}_{\mathrm{w}}=7,0 \mathrm{y} \mathrm{D}=$ $50 \mathrm{~km}$ que queda por debajo de la norma, ambos de origen cortical y estimados para suelo blando.

\section{Ecuaciones para GM PGA para eventos por subducción}

Para el caso de eventos generados por procesos de subducción, se decidió trabajar únicamente con sismos ocurridos en Costa Rica, debido a que son los que están mejor identificados y más claramente separados respecto a los de fallamientocortical, además de que con este grupo de datos fue con el que se obtuvo resultados más estables y congruentes al estimar los modelos para todo el rango de periodos seleccionados.

De acuerdo con el cuadro 4, 96 eventos de subducción generaron 267 registros acelerográficos, de los cuales solo un $12 \%$ corresponden a suelos tipo S I, un $37,8 \%$ a S II y un $50,2 \%$ a S III y S IV. Una vez más, se observa la poca cantidad de registros provenientes de emplazamientos en roca.

De acuerdo con las magnitudes $\mathrm{M}_{\mathrm{w}}$, un $58 \%$ de los datos tiene valores entre 4 y 4,9 , un $24,3 \%$ entre 5 y 5,9 y solamente un $17,7 \%$ tiene magnitudes superiores a 6,0 .

Respecto a la distribución gráfica de los datos, las figuras 13a y $13 \mathrm{~b}$ muestran las magnitudes en 



Fig. 11: Comparación entre los espectros estimados según distintos modelos. a) para suelo firme (S II) y b) para suelo blando (S III), con las propuestas por: Dahle et al. (1995), Schmidt et al. (1997) y Zhao et al. (2006a), para $\mathrm{M}_{\mathrm{W}}=6$ y D = $50 \mathrm{~km}$.

función de las distancias hipocentrales y se observan los puntos distribuidos en forma más o menos homogénea, con algunas zonas descubiertas, sobretodo para magnitudes alrededor de 6 y distancias intermedias, así como para $\mathrm{M}_{\mathrm{w}}=7$ y distancias hipocentrales cortas, entre 25 y $70 \mathrm{~km}$. Cabe mencionar que la distribución de datos no es tan densa como en el caso de sismos por fallamientocortical, analizados en la sección anterior.

Si se agrupan los datos según el tipo de suelo (Fig. 13b) se observan distribuciones poco uniformes para roca en todo el rango de magnitudes y
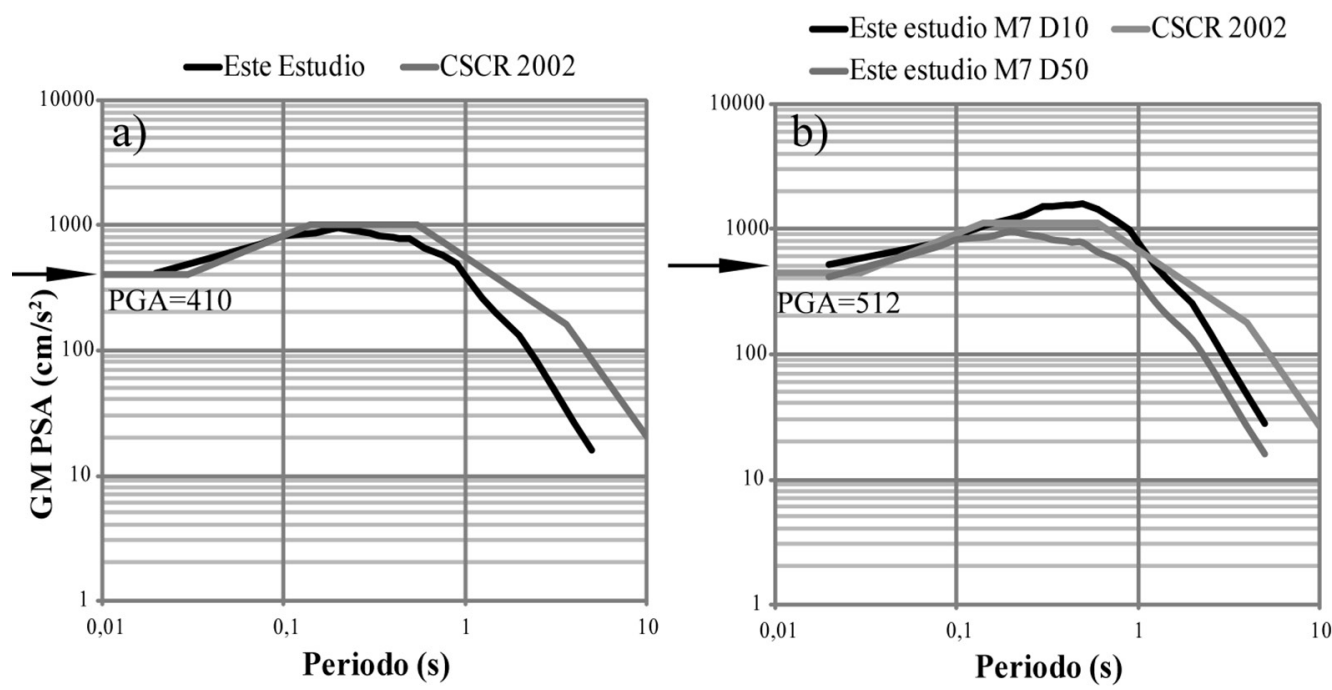

Fig. 12: Comparación de los espectros obtenidos a partir de las ecuaciones de atenuación con el Código Sísmico de Costa Rica (CSCR, 2010), para a) suelo firme y b) suelo blando. En ambos $\operatorname{casos} \mathrm{M}_{\mathrm{w}}=7 \mathrm{y} \mathrm{D}=10 \mathrm{~km}$. 
a)



b) sblando afirme $\Delta$ roca

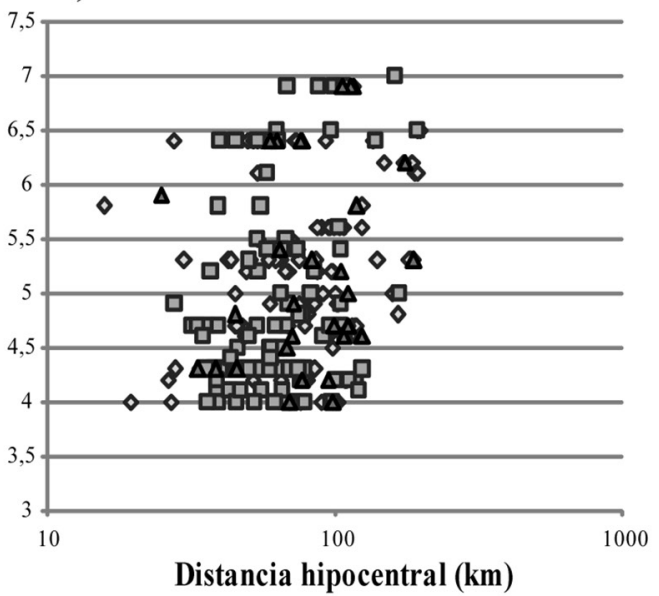

Fig. 13: Distribución de $M_{w}$ vs. distancia hipocentral (a) para sismos generados por subducción, Costa Rica y (b) separados según tipo de suelo para Costa Rica.

más o menos uniformes para suelos firme y roca, para magnitudes entre 5,5 y 6,5 . Entre 4 y 5,5, los datos se distribuyen en forma más uniforme para suelos firme y blando.

Las GM PGA se distribuyen respecto a la distancia hipocentral, tal y como lo muestran las figuras 14 a y $14 b$. Según el tipo de suelo, se observan datos distribuidos casi uniformemente en todo el rango de distancias y magnitudes, siendo más escasos para S I.
Si se considera la distribución de los datos respecto a los tres rangos de magnitudes (Fig. 14b), se evidencia la separación de los datos de acuerdo con ellos y la forma en que cada rango se atenúa con la distancia (líneas en distintos tonos de grises en la misma figura).

Después de realizar varios análisis con distintos tipos de ecuaciones, se plantea el modelo que tiene la siguiente forma:


Fig. 14: Distribución de GM PGA vs. distancia hipocentral, datos por subducción separados según tipo de suelo (a) y separados según rangos de magnitudes (b), datos de Costa Rica. 


\section{Cuadro 4}

Distribución de los datos de eventos por subducción provenientes de Costa Rica, de acuerdo al número de eventos, número de registros, tipo de suelo y rangos de magnitudes.

\begin{tabular}{cccc}
\hline Número de eventos & Número de registros & Según tipo de suelo & Según rango de magnitudes \\
\hline 96 & 267 & S I: $32(12 \%)$ & 4 a $4,9: 155(58 \%)$ \\
& & S II: $101(37,8 \%)$ & 5 a 5,9: $65(24,3 \%)$ \\
& S III y S IV: $134(50,2 \%)$ & 6 o más: $47(17,7 \%)$ \\
\hline
\end{tabular}

$$
\begin{array}{r}
\log \mathrm{Y}=\mathrm{C}_{1}+\mathrm{C}_{2} * \mathrm{M}+\mathrm{C}_{3} * \log \left(\operatorname{sqrt}\left(\mathrm{D}^{2}+5^{2}\right)\right)+\mathrm{S}^{*} \mathrm{C} 4 \\
+\mathrm{H}^{*} \mathrm{C}_{5}
\end{array}
$$

En este caso, el coeficiente correspondiente a la profundidad ficticia $\mathrm{b}_{4}$ se fijó a $5 \mathrm{~km}$ debido a que en muchos casos los métodos de inversión son incapaces de resolverlo, como sucedió aquí para algunos periodos.

El método utilizado corresponde a la regresión ordinaria de un paso (mínimos cuadrados), que también resultó ser el más estable. Los resultados se muestran en el cuadro 5 .

Los modelos obtenidos para distintas condiciones de suelo y GM PGA en función de la distancia hipocentral son mostrados en las figuras $15 \mathrm{a}, 15 \mathrm{~b}, 15 \mathrm{c}$ y $15 \mathrm{~d}$, donde los puntos negros corresponden a las observaciones ajustadas a las condiciones de suelo S I, S II y S III, respectivamente. Se dibujan además las curvas referentes a \pm 1 SD y se observa un adecuado ajuste visual de los datos dentro de estos rangos. Prácticamente no aparecen observaciones a distancias inferiores a los $25 \mathrm{~km}$ como era de esperarse, debido a la restricción impuesta a los datos de subducción.

Los residuos son mostrados en las figuras 16a en función de la distancia hipocentral y $17 \mathrm{~b}$ en función de la magnitud. Se observa en ambos casos que los residuos se ubican entre $-1 \mathrm{y}+1$, por lo que resultan ser inferiores y más cercanos a cero, respecto a los obtenidos para eventos por fallamiento cortical superficial.

Respecto a la magnitud, los residuos decrecen en función de esta y en ambos casos (distancia hipocentral y magnitud), la línea de residuo igual a cero coincide con la línea de tendencia, por lo que no hay un sesgo o inclinación de la misma.

Si se comparan las relaciones de atenuación obtenidas en el estudio para subducción con las de sismos por fallamiento cortical (Fig. 17a), se observa que las GM PGA para subducción son mayores, aún en el rango de validez de estos que es a partir de los $25 \mathrm{~km}$. La amplificación relativa entre S III y S II también es mayor para el caso de subducción que para fallamiento cortical ya que en este último, las amplificaciones relativas de $\mathrm{S}$ III a S II y de S II a S I son muy semejantes. Por otro lado, el decaimiento mostrado por las curvas para modelos por subducción es mayor.

Al comparar las ecuaciones predictivas del movimiento del suelo propuestas por distintos autores (Fig. 17b), se observa que la propuesta en este estudio es conservadora, ya que predice bajos valores a distancias hipocentrales cercanas a $25 \mathrm{~km}$ (línea vertical punteada en la misma figura), y se acerca a un promedio de las distintas propuestas a distancias mayores, cercanas al límite de $200 \mathrm{~km}$. Lo anterior significa que el modelo propuesto tiene un decaimiento menor con la distancia, que el resto de los modelos considerados, a excepción de los propuestos por Dahle et al. (1995) y Schmidt et al. (1997), que muestran un decaimiento aún menor y que por tanto, predicen mayores valores de GM PGA a distancias largas.

Esta comparación es importante ya que Schmidt et al. (1997) también obtuvieron ecuaciones de atenuación para sismos por subducción a partir de datos de Costa Rica, pero tal y como se

Cuadro 5

Coeficientes obtenidos de acuerdo con el modelo de inversión más estable para GM PGA, sismos por subducción.

\begin{tabular}{cccccc}
\hline $\mathrm{C} 1$ & $\mathrm{C} 2$ & $\mathrm{C} 3$ & $\mathrm{C} 4$ & $\mathrm{C} 5$ & $\mathrm{SD}$ \\
\hline 0,49807 & 0,5371 & $-1,30061$ & 0,35955 & 0,11626 & 0,352 \\
\hline
\end{tabular}





Fig. 15: Relaciones de atenuación obtenidas a partir de sismos por subducción registrados en Costa Rica, para $\mathrm{M}_{\mathrm{w}}=6$ a) para suelo S I, b) para suelo S II y c) para suelo S III. Las observaciones (puntos negros) se han ajustado a la respectiva condición de suelo. La figura d) compara las curvas obtenidas para los tres tipos de suelo considerados.

dijo antes, los resultados no corresponden al mismo grupo de datos ya que en 1997 predominaron los provenientes de instrumentos analógicos y los

La figura 18 compara las aceleraciones registradas en todas las estaciones del Laboratorio de Ingeniería Sísmica de la Universidad de Costa Rica para el terremoto de Sámara del 5 de setiembre de $2012\left(M_{w} 7,6\right)$, con las ecuaciones obtenidas en este estudio para subducción en suelo S II. A pesar de no haberse tomado este evento para generar las ecuaciones, es claro observar que todos los puntos observados se encuentran dentro del rango com- prendido entre el promedio \pm SD. Solamente se observa que a distancias lejanas (superiores a los 200 $\mathrm{km}$ ), las curvas no estiman apropiadamente la atenuación, debido a que a partir de esta distancia el término correspondiente a la atenuación inelástica no fue tomado en cuenta al plantear la ecuación 10.

\section{Ecuaciones para GM PSA para eventos por subducción}

Para las regresiones referentes a la media geométrica entre las dos pseudos aceleracio- 



Fig. 16: Residuos para eventos por subducción en función de la distancia hipocentral (a) y la magnitud (b).

nes espectrales horizontales (GM PSA), fueron considerados 23 periodos comprendidos entre 0,02 s y 5 s.Las ecuaciones predictivas estimadas para roca, $\mathrm{M}_{\mathrm{w}}=6$, así como los valores observados a partir de los cuales se obtuvieron las regresiones se muestran en la figura 19a. Nótese que en este caso, a diferencia del PGA, varios puntos negros (observaciones) se salen del rango definido por $\pm 1 \mathrm{SD}$. En la figura $19 \mathrm{~b}$ se puede notar la gran amplificación que se obtiene en el tipo de suelo S III y esto es debido al coeficiente $b_{4}=0,592$, que es muy superior al valor de $b_{5}=0,119$, que corresponde al suelo tipo S II, ambos casos comparados respecto a la roca (ver Cuadro 6, para $1 \mathrm{~s}$ ).

Respecto a los residuos de GM PSA para 1 $\mathrm{Hz}$, se observan valores mayores que los obtenidos para GM PGA. En el caso actual, algunos va-



Fig. 17: a) Comparación entre las relaciones de atenuación para sismos de fallamiento cortical y subducción obtenidas en el presente estudio, para las distintas condiciones de suelo consideradas. b) Comparación con las obtenidas por distintos autores, para eventos por subducción para $\mathrm{M}_{\mathrm{w}}=6$ y GM PGA. La línea vertical de puntos indica el inicio de la zona de validez para el modelo propuesto en este estudio, que corresponde a $25 \mathrm{~km}$. 


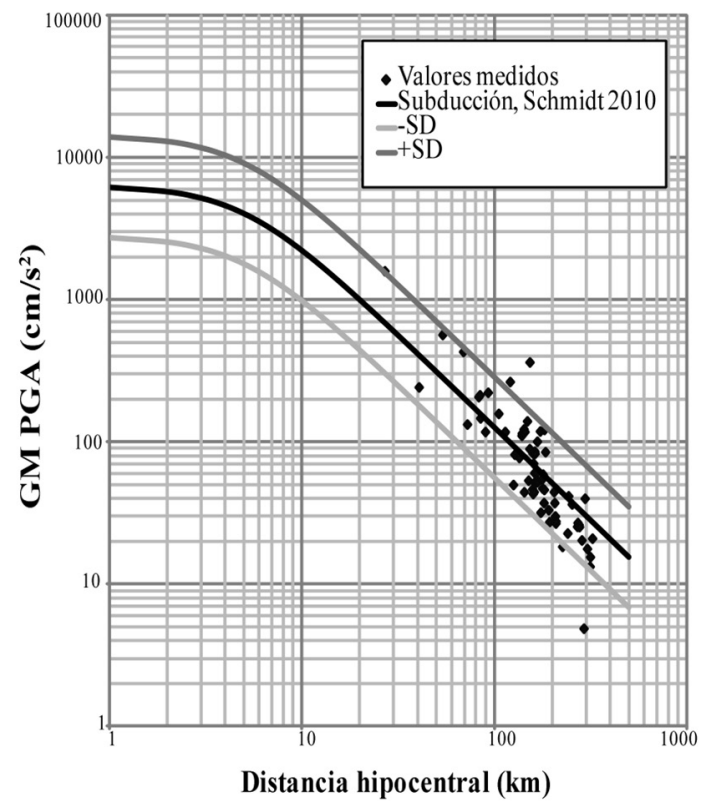

Fig. 18: Comparación entre aceleraciones registradas para el terremoto de Sámara (5/9/2012; $\left.M_{w} 7,6\right)$ con modelos de atenuación propuestos en esta investigación.

lores superan +1 y -1 de residuo pero la mayoría se concentra dentro de ese rango (Fig. 20a). En la figura 20b, donde se muestran los residuos en función de la magnitud, se observan valores altos para $\mathrm{M}_{\mathrm{w}}=5,6$, debido a que las observaciones superan a las estimaciones en buena medida. Si se analizan ambos gráficos, la línea promedio coincide con la del residuo igual a cero, por lo que no se evidencia un sesgo respecto al incremento de la distancia hipocentral o a la magnitud.

Si se comparan las ecuaciones obtenidas de GM PSA a $1 \mathrm{~Hz}$ para datos de sismos de fallamiento cortical con las de subducción (Fig. 21a), se observa que para estas últimas las ordenadas espectrales son mayores, sobretodo el efecto de amplificación por presencia de suelo S III es bastante mayor que para sismos de América Central por sismos corticales. El decaimiento de las curvas basadas en ambos modelos es parecido, ya que resultan ser bastante paralelas.

$\mathrm{Al}$ comparar las ecuaciones aquí propuestas con las de otros autores, sobretodo a partir del rango de validez de los datos del presente estudio $(25 \mathrm{~km}$ para subducción, definido por la línea vertical punteada en la figura 21b), se nota que los resultados para Costa Rica se asemejan mucho a los obtenidos por García et al. (2005) para registros en roca de sismos por subducción en México. Ambos casos predicen valores de GM PSA inferiores a casi todo el resto de los modelos considerados, aún cuando los demás decaen más
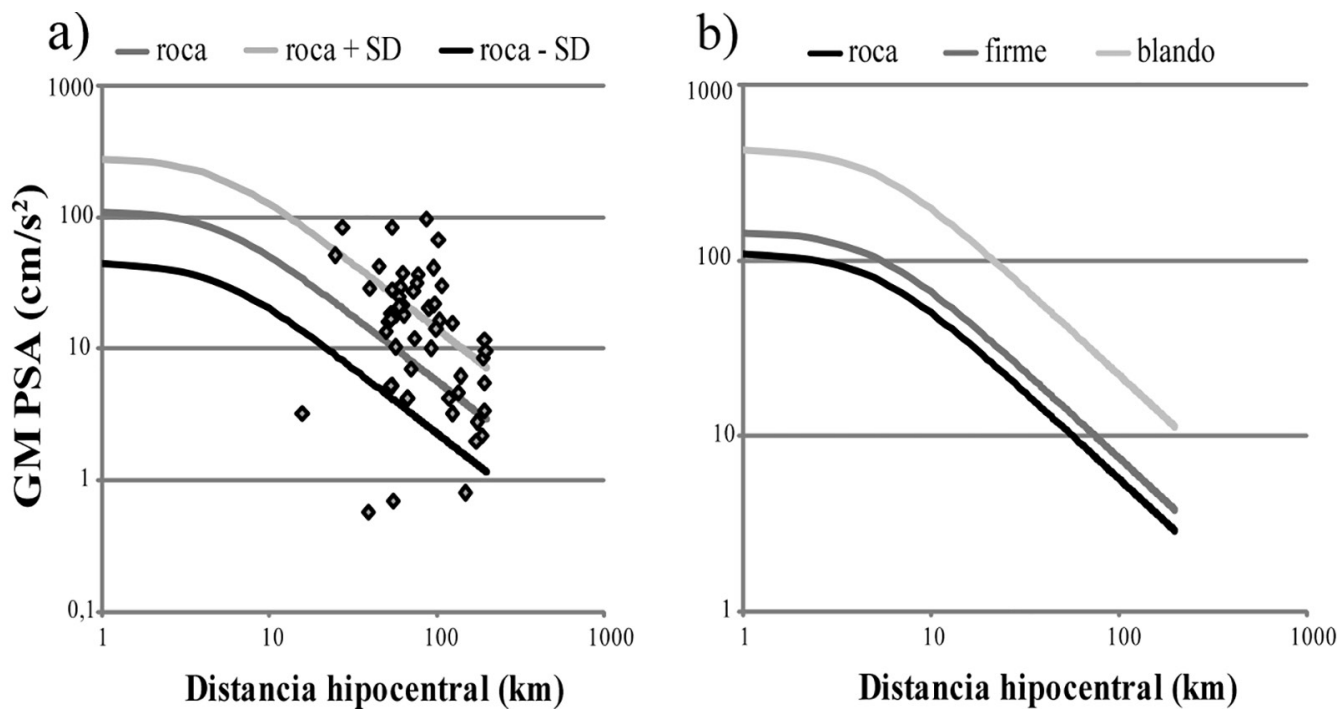

Fig. 19: Resultados obtenidos con los datos de subducción para Costa Rica. La figura a) muestra las observaciones (puntos negros) transformadas a roca y los rangos que definen $\pm 1 \mathrm{SD}$. La figura $\mathrm{b}$ ) muestra las curvas obtenidas para los distintos tipos de suelo. 

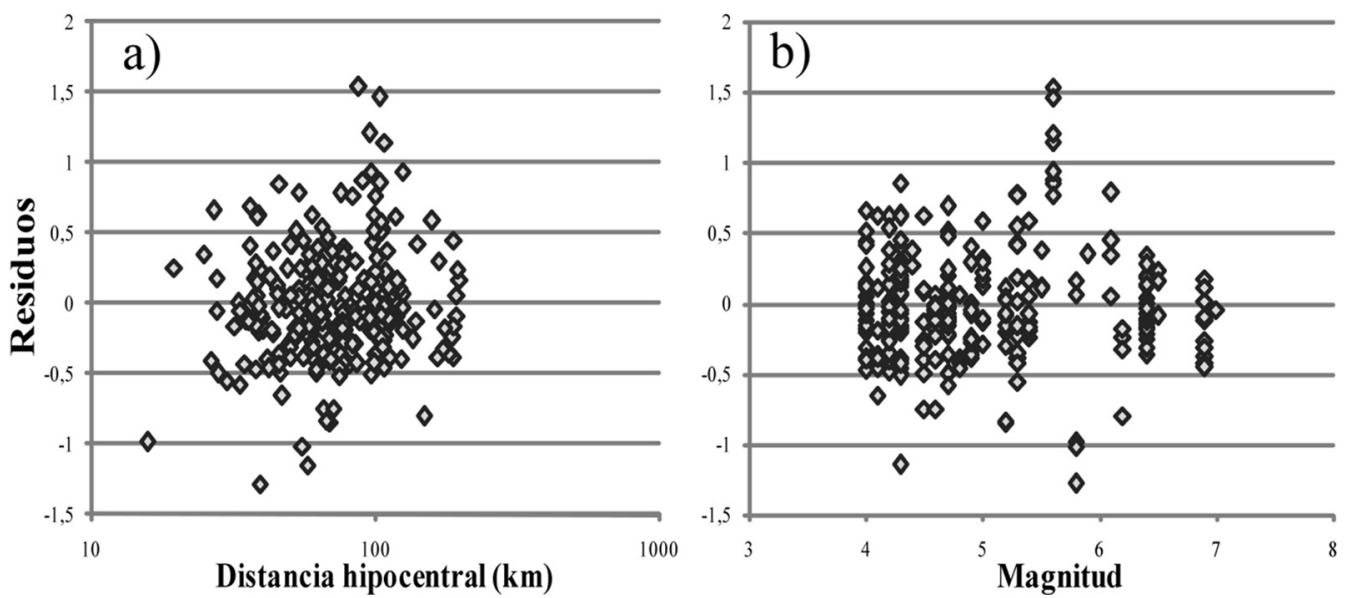

Fig. 20: Residuos obtenidos a partir de las ecuaciones de atenuación para subducción de Costa Rica, en función de la distancia hipocentral (a) y en función de la magnitud (b).

Cuadro 6

Coeficientes y desviaciones estándar para 23 periodos seleccionados. Sismos de subducción de Costa Rica.

\begin{tabular}{|c|c|c|c|c|c|c|c|}
\hline Period & frec. & $\mathrm{C} 1$ & $\mathrm{C} 2$ & $\mathrm{C} 3$ & $\mathrm{C} 4$ & $\mathrm{C} 5$ & SD \\
\hline 0,02 & 50,00 & 0,525 & 0,536 & $-1,308$ & 0,358 & 0,119 & 0,353 \\
\hline 0,04 & 25,00 & 0,732 & 0,524 & $-1,365$ & 0,345 & 0,127 & 0,352 \\
\hline 0,075 & 13,33 & 1,227 & 0,496 & $-1,465$ & 0,299 & 0,134 & 0,355 \\
\hline 0,1 & 10,00 & 1,292 & 0,486 & $-1,403$ & 0,252 & 0,115 & 0,349 \\
\hline 0,15 & 6,67 & 1,137 & 0,505 & $-1,294$ & 0,253 & 0,034 & 0,368 \\
\hline 0,2 & 5,00 & 0,75 & 0,544 & $-1,215$ & 0,311 & 0,079 & 0,362 \\
\hline 0,24 & 4,17 & 0,34 & 0,585 & $-1,168$ & 0,411 & 0,122 & 0,368 \\
\hline 0,303 & 3,30 & $-0,081$ & 0,623 & $-1,146$ & 0,58 & 0,168 & 0,361 \\
\hline 0,34 & 2,94 & $-0,291$ & 0,648 & $-1,144$ & 0,622 & 0,173 & 0,372 \\
\hline 0,4 & 2,50 & $-0,67$ & 0,678 & $-1,082$ & 0,651 & 0,172 & 0,371 \\
\hline 0,44 & 2,27 & $-0,882$ & 0,696 & $-1,066$ & 0,689 & 0,186 & 0,373 \\
\hline 0,5 & 2,00 & $-1,148$ & 0,739 & $-1,087$ & 0,696 & 0,19 & 0,373 \\
\hline 0,6 & 1,67 & $-1,471$ & 0,787 & $-1,094$ & 0,649 & 0,134 & 0,373 \\
\hline 0,752 & 1,33 & $-1,914$ & 0,842 & $-1,108$ & 0,632 & 0,135 & 0,387 \\
\hline 0,9 & 1,11 & $-2,333$ & 0,868 & $-1,038$ & 0,617 & 0,132 & 0,396 \\
\hline 1 & 1,00 & $-2,555$ & 0,882 & $-0,991$ & 0,592 & 0,119 & 0,397 \\
\hline 1,25 & 0,80 & $-2,925$ & 0,898 & $-0,908$ & 0,529 & 0,113 & 0,401 \\
\hline 1,493 & 0,67 & $-3,3$ & 0,932 & $-0,889$ & 0,527 & 0,106 & 0,412 \\
\hline 2 & 0,50 & $-3,706$ & 0,964 & $-0,909$ & 0,53 & 0,13 & 0,412 \\
\hline 2,5 & 0,40 & $-3,927$ & 0,981 & $-0,921$ & 0,488 & 0,135 & 0,398 \\
\hline 3,03 & 0,33 & $-3,954$ & 0,979 & $-0,973$ & 0,439 & 0,124 & 0,386 \\
\hline 4 & 0,25 & $-3,943$ & 0,944 & $-0,981$ & 0,376 & 0,108 & 0,384 \\
\hline 5 & 0,20 & $-3,954$ & 0,923 & $-0,989$ & 0,314 & 0,112 & 0,38 \\
\hline
\end{tabular}



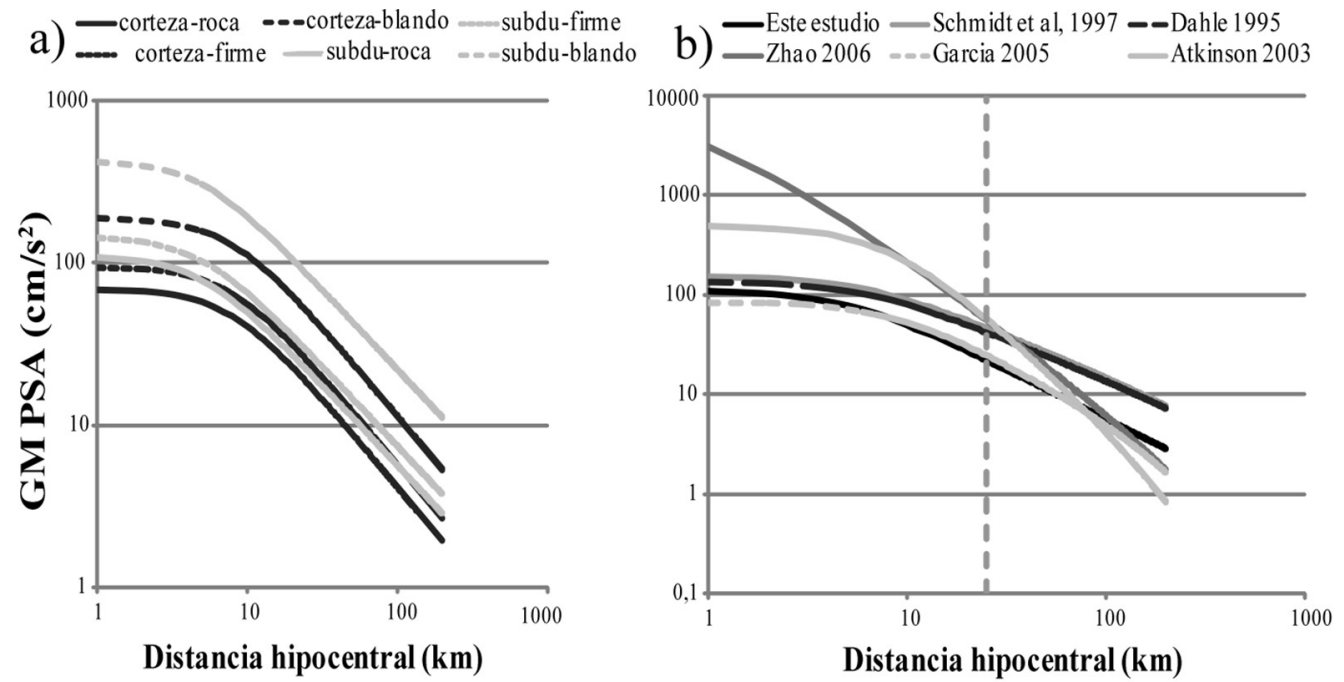

Fig. 21: a) Comparación entre modelos de sismos por subducción (líneas grises) y de fallamiento cortical (líneas negras) según el tipo. b) Comparación de los resultados obtenidos en este estudio con los propuestos por otros autores, para $1 \mathrm{~Hz}$ GM PSA, para suelo $\mathrm{S}$ I y $\mathrm{M}_{\mathrm{w}}=6$. según el tipo de suelo, para $\mathrm{M}_{\mathrm{w}}=6$ y $1 \mathrm{~Hz}$ de PSA. de suelo, para $\mathrm{M}_{\mathrm{w}}=6$ y $1 \mathrm{~Hz}$ de PSA.

fuertemente con la distancia (Atkinson \& Boore, 2003; Zhao et al., 2006a).

Hay una clara diferencia con los modelos propuestos tanto por Dahle et al. (1995) como por Schmidt et al. (1997), ya que estos predicen valores mayores en todo el rango de periodos y tienen un decaimiento menor con la distancia.

El cuadro 6 muestra los coeficientes y las desviaciones estándar correspondientes a cada periodo seleccionado. Estos son representados gráficamente en las figuras 22a, 22b, 22c y 22d.

Se observa como $\mathrm{C} 1$, que se relaciona con la fuente generadora del sismo, predominantemente decae con el aumento del periodo, sobretodo entre $0,1 \mathrm{~s}$ y $2,5 \mathrm{~s}$, de un valor de $+1,29$ a -4 (Fig. 22a). Sin embargo, muestra un comportamiento esperado y semejante al de sismos corticales, siendo mayores los obtenidos con sismos por subducción.

Por el contrario $\mathrm{C}_{2}$ (coeficiente de escala de magnitud, figura 22b) aumenta con el periodo, de 0,48 en 0,1 s a 0,979 en $3 \mathrm{~s}$. Este coeficiente se comporta en forma bastante semejante al b2 para sismos corticales de América Central y es coherente con el hecho de que la magnitud tiene mayor influencia en periodos largos.
El coeficiente $\mathrm{C}_{3}$, que representa la expansión geométrica o atenuación en función de la distancia, tiene una variación mayor y valores algo superiores que para sismos de fallamientocortical, incrementándose en este caso de $-1,4$ en $0,1 \mathrm{~s}$ a $-0,9$ en $1,25 \mathrm{~s}$, para luego sufrir un descenso hasta llegar a un valor de -1 (Fig. 22c).

En la figura $22 \mathrm{~d}$ se observa que $\mathrm{C}_{5}$ es bastante estable debido a que tiene un valor promedio cercano a 1,3 en todo el rango de periodos. Sin embargo, $\mathrm{C} 4$ es bastante más alto y para un periodo de 0,5 s llega a un valor de 5 .

Este efecto de amplificación excesiva por presencia de suelos blandos puede deberse a que los suelos en América Central en general tienen un grado mayor de meteorización y de más mala calidad que otras latitudes debido a que son tierras más jóvenes formados en ambientes tropicales?.

Por otro lado, la desviación estándar SD tiene un ligero incremento gradual con el aumento del periodo, desde 0,35 en $0,02 \mathrm{~s}$ hasta 0,41 en 1,5 s. Estos valores son algo inferiores a los obtenidos con sismos por fallamiento cortical para América Central, pero su tendencia al incremento es similar.

Las figuras 23a y 23b comparan los espectros para GM PSA obtenidos para sismos por 

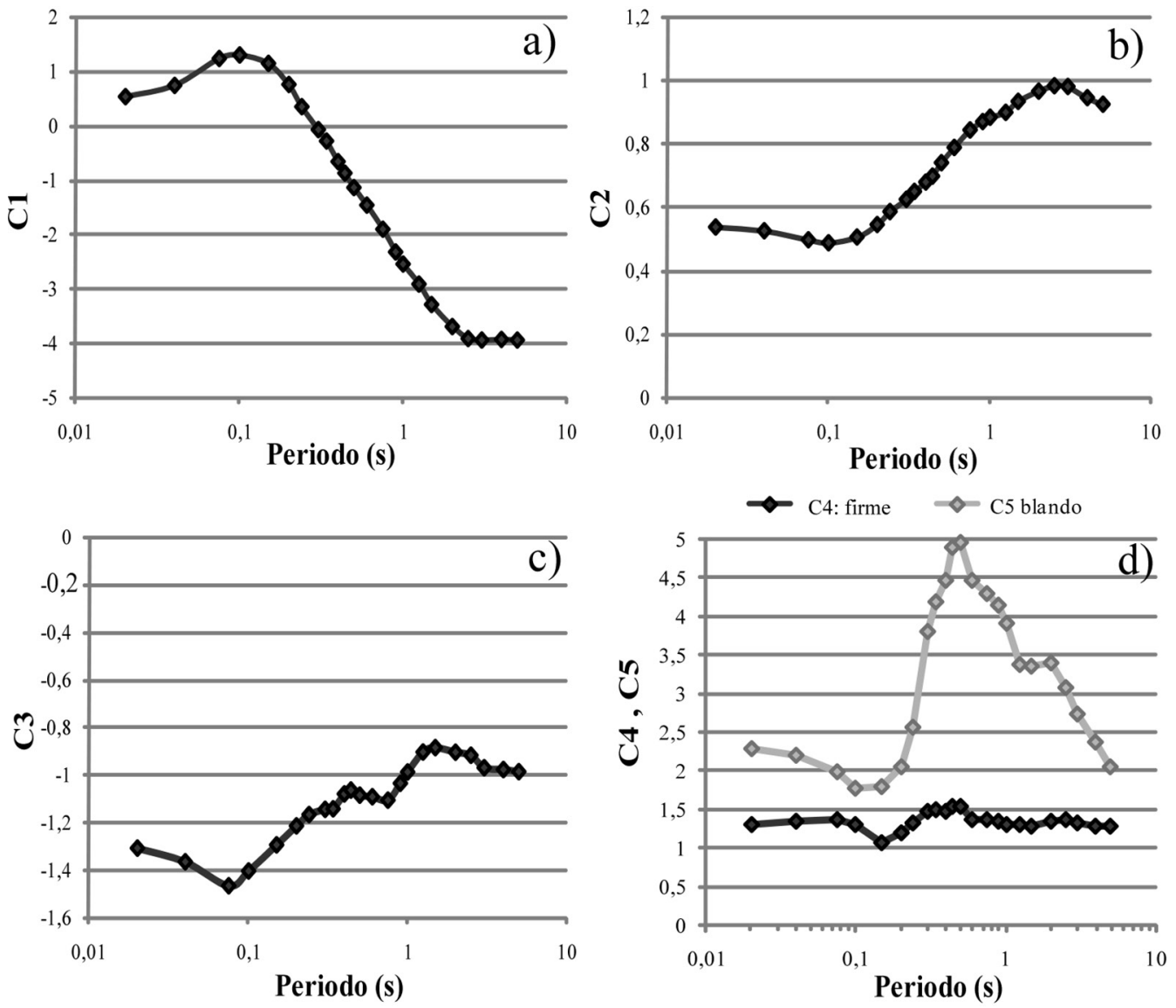

Fig. 22: Representación gráfica de los coeficientes $C_{1}$ a $C_{3}$ y de las desviaciones estándar mostrados en el cuadro 6 para sismos por subducción de Costa Rica.

fallamiento cortical (América Central) y por subducción (Costa Rica), para registros en roca y dos distancias hipocentrales distintas; 50 y $100 \mathrm{~km}$. Se observan formas espectrales parecidas, en ambos casos se evidencia un pico en $0,15 \mathrm{~s}$ y se nota cómo para magnitudes más altas, los espectros se vuelven más suavizados y horizontales. Para todo el rango de periodos, los espectros de sismos de subducción tienen ordenadas espectrales mayores que los de fallamiento cortical.

$\mathrm{Al}$ comparar los espectros propuestos por diversos autores con los obtenidos en el presente estudio para $\mathrm{M}_{\mathrm{w}}=6 \mathrm{y}$ distancia hipocentral de $50 \mathrm{~km}$, se observa que para roca (figura 24a) este estudio coincide bastante bien con el de García et al. (2005) a partir de los 0,3 s de periodo. Sin embargo, en el rango de 0,1 a $0,3 \mathrm{~s}$ los modelos para
Costa Rica predicen mayores ordenadas espectrales y para periodos inferiores a $0,1 \mathrm{~s}$ esta situación se invierte, resultando mayores las de México.

Zhao et al. (2006a) se mantiene siempre por encima de todos los modelos, así como sucede con los de Dahle et al. (1995) y Schmidt et al. (1997), que son considerablemente mayores a los que aquí se proponen a partir de $0,24 \mathrm{~s}$.

Si se analiza el gráfico para las mismas condiciones de distancia y magnitud anteriores pero para suelo blando (figura 24b), se observa que los valores de las ordenadas espectrales del presente estudio superan las propuestas por los otros autores, desde $0,1 \mathrm{~s}$ hasta $0,75 \mathrm{~s}$. A partir de ese periodo las curvas se asemejan, teniendo un decaimiento menor (mayores valores de GM PSA) las propuestas por Dahle et al. (1995) y Schmidt et al. 

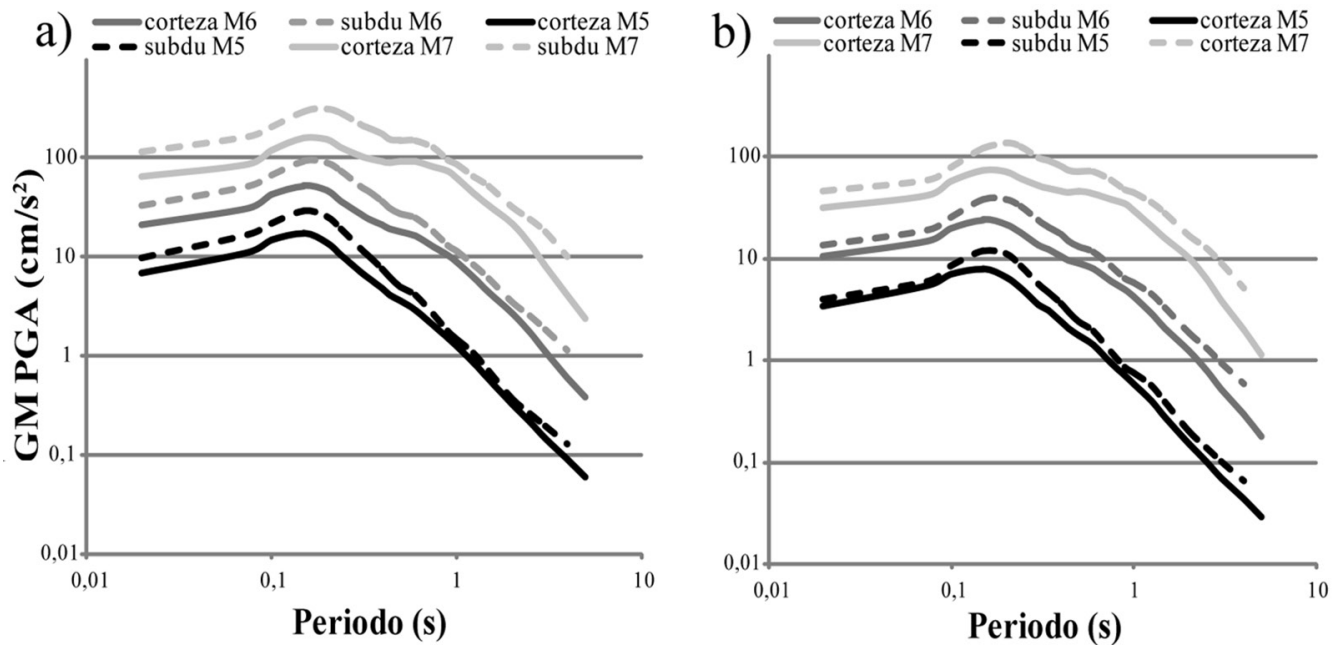

Fig. 23: Comparación entre espectros de sismos de fallamiento cortical (líneas continuas) y de subducción (líneas discontinuas), para magnitudes $M_{w}$ de 5, 6 y 7, suelo tipo S I y distancias hipocentrales de $50 \mathrm{~km}$ (a) y de $100 \mathrm{~km}$ (b).

(1997). Obsérvese que no se compara con García et al. (2005) debido a que para esas ecuaciones solo se consideraron datos para roca.

La amplificación descrita en el párrafo anterior es debida a los altos valores obtenidos para el coeficiente b4 (para suelo blando, S III) según el cuadro 6 y la figura $22 \mathrm{~d}$, situación que refuerza la idea de que los suelos blandos en el área de estudio provocan una fuerte amplificación para periodos mayores a $0,1 \mathrm{~s}$.

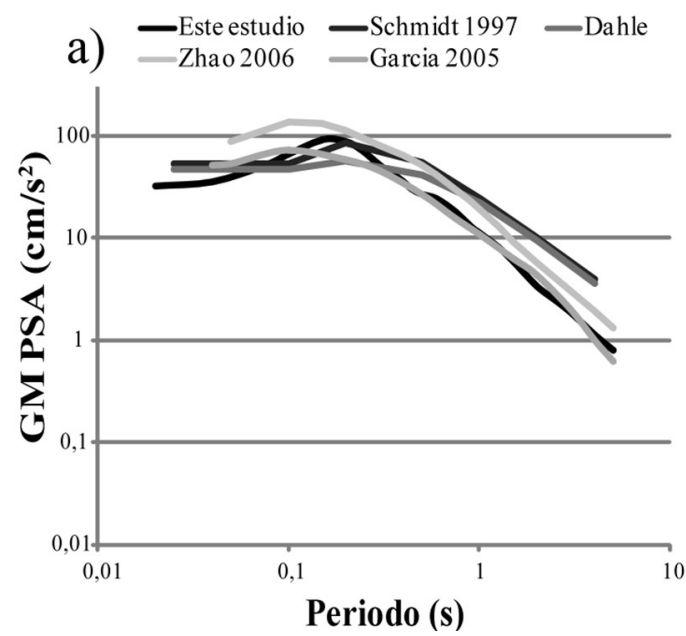

Finalmente, la figura 25 compara los espectros obtenidos a partir de las ecuaciones propuestas para un sismo de magnitud $\mathrm{M}_{\mathrm{w}}=7 \mathrm{y}$ distancia hipocentral $\mathrm{D}=25 \mathrm{~km}$ con los espectros de diseño del Código Sísmico de Costa Rica (CFIA, 2002), para la ciudad de San José (Zona III), para roca (Fig. 25a) y suelo blando (Fig. 25b).

Se observa que para roca, el espectro de la norma se ubica por encima del obtenido con las ecuaciones para esos dos parámetros de magnitud

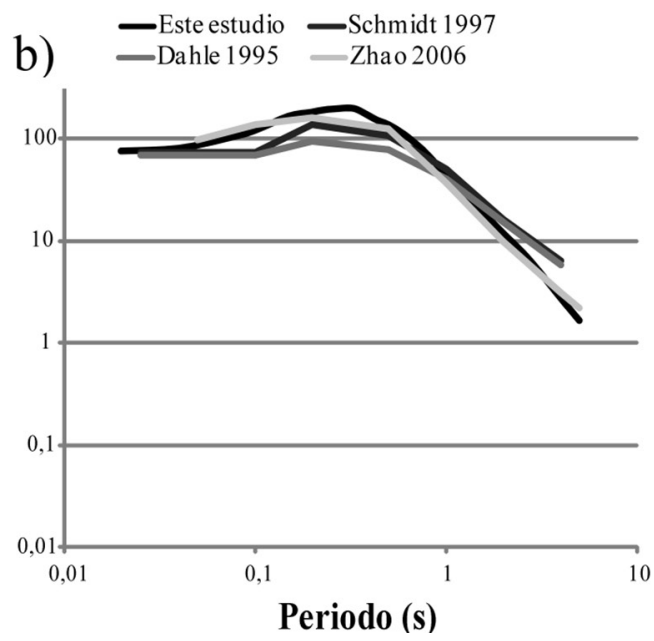

Fig. 24: Espectros de respuesta que comparan los resultados obtenidos en el presente estudio con los propuestos por diversos autores para $\mathrm{M}_{\mathrm{w}}=6 \mathrm{y}$ una distancia hipocentral de $50 \mathrm{~km}$. La figura a) corresponde a roca y la b) a suelo blando. 

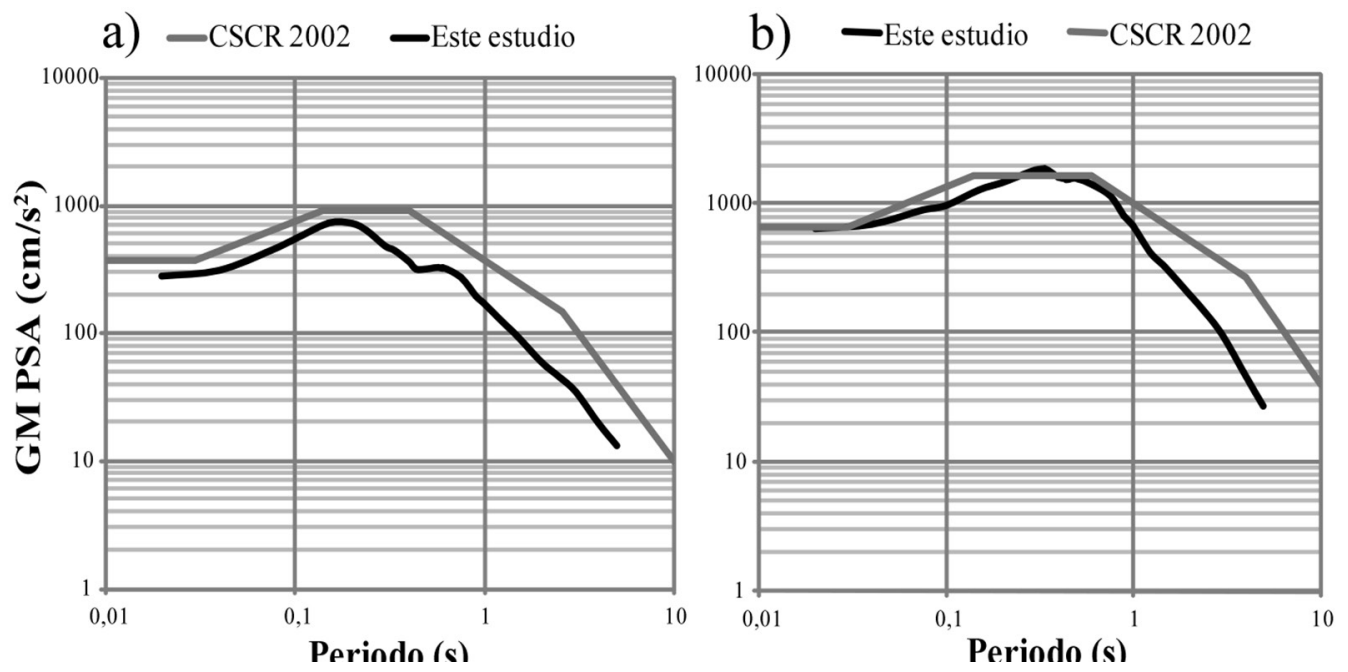

Fig. 25: Comparación entre los espectros del Código Sísmico de Costa Rica con los obtenidos en este estudio para un sismo de $\mathrm{M}_{\mathrm{w}}$ $=7,0$ y D $=25 \mathrm{~km}$, para a) roca, b) suelo blando.

y distancia, por lo que se puede afirmar que de acuerdo con los resultados obtenidos, el espectro que propone la norma cubre al menos a los sismos por subducción, con magnitud de 7 y distancia hipocentral cercana a $25 \mathrm{~km}$. Por lo tanto, sismos con este origen con magnitudes inferiores a 7 y distancias superiores a $25 \mathrm{~km}$ están cubiertos también.

Al comparar los espectros del código mencionado con los resultados obtenidos en esta investigación para suelo blando, se observa al igual que en el caso de sismos corticales, que a partir de $1 \mathrm{~s}$ de periodo, el espectro de la norma es conservador respecto al espectro propuesto aquí, pero ambos son muy semejantes desde $0,01 \mathrm{~s}$ hasta $1 \mathrm{~s}$.

\section{CONCLUSIONES}

Se obtuvieron modelos de predicción del movimiento del suelo, también conocidos como ecuaciones del atenuación, con datos registrados en América Central, provenientes principalmente de Costa Rica, El Salvador y Nicaragua.

Para el caso de sismos de origen cortical, se trabajó con datos provenientes de Nicaragua, El Salvador y Costa Rica y las ecuaciones se obtuvieron tanto para GM PGA como para 22 frecuencias de GM PSA.
En todas las soluciones propuestas, en general los coeficientes obtenidos tienen un comportamiento estable y similar en todo el rango de periodos y su variación concuerda con el proceso que a cada uno de ellos le corresponde cumplir dentro de la ecuación.

La desviación estándar (SD) obtenida para GM PGA es alta de acuerdo con resultados obtenidos en otras partes del mundo y aumenta para GM PSA, como es lo usual debido en alguna medida a los procesos de registro y digitalización que influyen más en frecuencias bajas.

Al comparar los resultados de GM PGA con los obtenidos por otros autores (Dahle et al., 1995, Schmidt et al., 1997, Zhao et al., 2006a, Spudich et al., 1999), se observa que los aquí propuestos son inferiores en casi todo el rango de distancias. Esto puede estar asociado al uso del método de mínimos cuadrados que fue el considerado en este caso, ya que fue el único que permitió obtener regresiones sin desestabilizarse. En el caso de los modelos propuestos para la misma región por otros autores, debe destacarse que el grupo de datos utilizado es distinto ya que en el pasado se usaron registros analógicos y en el presente, el 95\% de los registros fueron obtenidos por instrumentos digitales. 
Para el caso de GM PSA $1 \mathrm{~Hz}$, los efectos de sitio, representados por el coeficiente $\mathrm{C}_{5}$ que corresponde a suelo S III, es mayor que para GM PGA como era de esperarse, ya que la amplificación es más grande para frecuencias bajas (periodos largos). Para esta misma inversión, los residuos no muestran ningún sesgo en función de la distancia o de la magnitud y son menores que los obtenidos para GM PGA.

Si se comparan los espectros obtenidos con base en este estudio para $\mathrm{M}_{\mathrm{w}}=6 \mathrm{y} \mathrm{D}=50 \mathrm{~km}$ para los tres tipos de suelo considerados, se evidencia que las ordenadas aquí obtenidas son inferiores a las mostradas por otros autores en todo el rango de periodos para la condición de roca. Para suelo S III, hay una semejanza mucho mayor para todas las ordenadas espectrales y esto se debe principalmente a los altos factores de amplificación obtenidos en el presente estudio para esta condición de suelo.

Cuando se comparan los espectros obtenidos en esta investigación para sismos corticales de América Central para $\mathrm{M}_{\mathrm{w}}=7,0$, distancia hipocentral de $10 \mathrm{~km}$ y las tres condiciones de suelo consideradas, con los propuestos por el Código Sísmico de Costa Rica (CSCR-2002) para un periodo de retorno de 500 años en zona III, que es donde se concentra la mayor cantidad de población del país, se evidencia que en roca, todas las ordenadas espectrales de la norma se ubican por encima del espectro propuesto en esta investigación. Para suelo firme, ambos espectros son muy semejantes a excepción del fuerte decaimiento que muestra el espectro obtenido con las ecuaciones predictivas a partir de $1,0 \mathrm{~s}$ de periodo. Para suelo blando, el espectro de la norma se ubica entre dos sismos, ambos de $\mathrm{M}_{\mathrm{w}}=7,0$ pero con distancias hipocentrales de 10 y $50 \mathrm{~km}$, mostrando los del código una vez más un decaimiento más suave a partir de un periodo de $1,0 \mathrm{~s}$.

Para el caso del análisis de eventos por subducción, se seleccionaron solamente los datos de Costa Rica, debido a que son los que se tienen mejor identificados y más claramente separados respecto a los de fallamiento cortical. Además, con este grupo de datos es con el que se obtuvo resultados más congruentes y estables. Se trabajó tanto para GM PGA como para 23 frecuencias de GM PSA.

Fueron utilizados 276 registros provenientes de 96 sismos por subducción, de los cuales un $12 \%$ corresponde a suelo SI, un $37 \%$ a S II y un $50,2 \%$ a S III y a S IV. Una vez más, se observa la poca cantidad de registros provenientes de roca.

La mayor parte de los datos (un 58\%) corresponde a $\mathrm{M}_{\mathrm{w}}$ entre 4 y 4,9 y solamente un $17,7 \%$ a $\mathrm{M}_{\mathrm{w}}>6$. La distribución de $\mathrm{M}_{\mathrm{w}}$ vs. distancia hipocentral no es tan densa como en el caso de sismos por fallamiento cortical para América Central, sobretodo para magnitudes alrededor de 6 .

Después de realizar varias pruebas, los resultados más estables y con menor desviación estándar fueron obtenidos para el mismo tipo de ecuación seleccionado para sismos por fallamiento cortical, solamente que la distancia ficticia es fijada a $5 \mathrm{~km}$, lo que permitió obtener resultados estables para todas las frecuencias. El tipo de regresión utilizado es el de mínimos cuadrados (ordinario de un paso).

Se evidencia una mayor amplificación relativa de S III (representado por b4) respecto a S II (representado por $\mathrm{b}_{5}$ ) que de S II respecto a S I (roca).

$\mathrm{Si}$ se comparan con las curvas propuestas para fallamiento cortical de América Central, se observan mayores GM PGA y un decaimiento mayor de las curvas para eventos por subducción.

Al comparar con los modelos de otros autores, el aquí propuesto da valores más bajos a distancias cortas y a distancias más lejanas (cercanas al límite considerado de $200 \mathrm{~km}$ ), se vuelve muy semejante al promedio de todos los considerados para GM PGA.

Las diferencias observadas con respecto al otro modelo propuesto para Costa Rica en 1997, pueden deberse a que el grupo de datos es distinto a pesar de provenir de un mismo país, ya que en el modelo actual solo se usaron registros digitales.

Al obtener los modelos para GM PSA $1 \mathrm{~Hz}$, se evidencia la gran amplificación que se tiene con S III respecto a S II. Esto es evidente tam- 
bién si se compara con los resultados obtenidos por fallamiento cortical para América Central, donde los coeficientes que representan a S III son menores.

Si se observan los espectros de respuesta obtenidos para un amplio rango de periodos, lo más sobresaliente es la gran amplificación de S III respecto a S II en todo ese rango y que S II amplifica relativamente poco respecto a la roca.

Con respecto a los coeficientes $\mathrm{C}_{1}$ y $\mathrm{C}_{2}$, se comportan más o menos en forma semejante tanto para fallamiento cortical como para subducción. $\mathrm{C}_{3}$ es algo más estable (menos variable) para sismos por fallamiento cortical y la SD es algo menor para sismos por subducción que para sismos corticales, en todo el rango de frecuencias.

Al compararse los resultados de la presente investigación con las de otros autores (Dahle et al., 1995, Schmidt et al., 1997, Zhao et al., 2006a, García et al., 2005) se observa que para roca, hay una coincidencia bastante buena con la propuesta con datos de México y ambas resultan inferiores a las demás. Sin embargo, esta situación se invierte para suelo S III ya que, debido a los grandes factores de amplificación obtenidos para este tipo de suelo para datos de Costa Rica en casi todo el rango de frecuencias, estos espectros superan a los demás hasta llegar a 1,0 $\mathrm{s}$ de periodo.

Los espectros obtenidos en esta investigación para sismos por subducción con datos de Costa Rica, para $\mathrm{M}_{\mathrm{w}}=7,0$ y distancia hipocentral de 25 $\mathrm{km}$ se ubican por debajo de los propuestos por el Código Sísmico de Costa Rica (CSCR-2002) para zona sísmica III y roca. Si se comparan para suelo blando, se tiene un muy buen ajuste mostrando los espectros obtenidos en este trabajo un decaimiento más fuerte a partir de 1,0 $\mathrm{s}$ de periodo.

\section{REFERENCIAS BIBLIOGRÁFICAS}

\author{
ATAKAN, K., BARD, P-Y., KIND, F., MORENO, \\ B., ROQUETTE, P., TENTO, A. \& THE \\ SESAME TEAM, 2004: A standardized \\ software solution for the H/V spectral ra-
}

tio technique.- $13^{\text {th }}$ World Conference on Earth. Eng. Vancouver, Canada, paper No. 2270: 1-12.

ATKINSON, A. \& BOORE, D., 2003: Empirical ground-motion relations for subductionzone earthquakes and their applications to Cascadia and other regions.- Bull. Seism. Soc. Am. 93(4): 1703-1729.

BEYER, K. \& BOMMER, J., 2006: Relationships between median values and between aleatory variabilities for different definitions of the horizontal component of motion.- Bull. Seism. Soc. Am. 96(4): 1512-1522.

BOORE, D.M. \& JOYNER, W.B., 1982: The empirical prediction of ground motion.- Bull. Seism. Soc. Am. 72(6): s43-s60.

BUNDSCHUH, J. \& ALVARADO, G. (ed.), 2007: Central America: Geology, resources and hazards (vol. 1 y 2).- 1311 págs. Taylor \& Francis, The Netherlands.

CAMPBELL , K., 2003: A contemporary guide to strong-motion attenuation relations. in: international handbook of earthquake and engineering seismology. Part B-, Academic Press, London [CD-ROM Suplemento al capítulo 60, vol 2].

CLIMENT, A., BUNGUM, H., CIUDADREAL, M., DAHLE, A., SANTANA, G., STRAUCH, W., TAYLOR, W. \& VILLAGRÁN, M., 1994: Spectral strong motion attenuation in Central America.- 46 págs. NORSAR, Norway [Reporte técnico No. 2-17].

DAHLE, A., CLIMET, A., TAYLOR, W., \& BUNGUM, H., 1995: New spectral strong motion attenuation models for Central America. Proceedings of the 5th Int. Conference on Seismic Zonation, Vol II:1005-1012. 
DESHON, H., BILEK, S., DIXON, T., DORMAN, L., FLUEH, E., GONZALEZ, V., PROTTI, J., \& SCHWARTZ, S., 2003: Seismogenic zone structure of the southern Middle America Trench, Costa Rica. J Geophys. Res. Vol. 108, No. B10, 2491.

DOUGLAS, J., 2004: Ground motion estimation equations 1964-2003, (reissue of Report No. 01-1).- 239 págs. Imperial College of London, Londres [Reporte de Investigación No. 04-001-SM.]

GARCÍA, D., HERRAIZ, M., ORDAZ, M., PACHECO, J.F. \& SINGH, S.K., 2005: Inslab earthquakes of central México: peak ground-motion parameters and response spectra.- Bull. Seism. Soc. Am. 95(6): 2272-2282.

GÜENDEL F. \& PROTTI M., 1998: Sismicidad y sismotectónica de América Central.- Física de la Tierra, 10: 19-51.

HERMANN, R. \& KIJKO, A., 1983: Short-period Lg magnitudes: instrument, attenuation and source effects.- Bull. Seism. Soc. Am. 73(64): 1835-1850.

JOYNER, W.B. \& BOORE, D.M., 1981: Peak horizontal acceleration and velocity from strong-motion records including records from the 1979 Imperial Valley, California earthquake.- Bull. Seism. Soc. Am. 71:2011-2038.

JOYNER, W.B., \& BOORE, D.M., 1993: Methods for regression analysis of strong-motion data.- Bull. Seism. Soc. Am. 83:469-487.

LAPORTE, M., 2006:Correlación entre la aceleración pico y la aceleración pico efectiva y valores de PGV/PGA y PGA*PGD/PGV2 del banco de datos de movimientos fuertes de América Central.- IX Seminario Nac. de Geotécnia, San José, Costa Rica, 1:21.

MONTERO, W., PERALDO, G. \& ROJAS, W., 1997: Proyecto: amenaza sísmica de América Central,- 79 págs. Escuela Centroamericana de Geología, San José [Inf. Final].

NORABUENA, E., DESHON, H., DIXON, T., DORMAN, L., FLUEH, E., GONZALEZ, V., LUNDGREN, P., NEWMAN, A., POLLITZ, F., PROTTI, M., SAMPSON, D. \& SCHWARTZ, S., 2004: Geodetic and seismic constrains on some seismogenic zone processes in Costa Rica.J. Geophy. Research. 109, B11403, DOI:10.1029/2003JB002931.

OKAL, E.A. \& ROMANOWICZ, B.A., 1994: On the variation of b-values with earthquake size.- Phys. Earth Planet. Inter. 87: 55-76.

QUINTERO, R. \& GÜENDEL, F., 2000: Stress field in Costa Rica, Central America.Journal of Seismology, 4: 297-319.

ROJAS, W., BUNGUM, H. \& LINDHOLM, C., 1993: A catalog of historical and recent earthquakes in Central America.- 77 págs. Project Reduction of Natural Disasters in Central America, NORSAR, Norway [Reporte técnico No. 2-7]

ROJAS, W., ALVARADO, G., BARQUERO, R., BOSCHINI, I., BUNGUM, H., CLIMENT, A., ESQUIVEL, L., FERNÁNDEZ, M., LINDHOLM, C., MONTERO, W., MOYA, A., PROTTI, M., SCHMIDT, V. \& SOTO, G., 1998: Seismic hazard analy- 
sis for the metropolitan area of the Central Valley, Costa Rica.- 45 págs. NORSAR, Noruega [Reporte Técnico].

SALLARES, V., DAÑOBEITIA, J. J. \& FLUEH, E.R., 2000: Seismic tomography with local earthquakes in Costa Rica.- Tectonophysics 329:61-78.

SCHMIDT, V., BUNGUM, H. \& DAHLE, A., 1997: Costa Rican spectral strong motion attenuation.- 45 págs. NORSAR, Norway [Reporte Técnico].

SCHMIDT, V., 2010: Avances para estudios de riesgo sísmico a escala regional y local: aplicaciones a América Central y la Bahía de Cádiz (sur de España).- 307 págs. Univ. Politécnica de Cataluña, Barcelona [Tesis Ph.D].

SCHMIDT, V., 2011a: Clasificación de suelos basada en el cálculo de razones espectrales en sitios donde se ubican estaciones acelerográficas de América Central, casos de: El Salvador, Nicaragua y Costa Rica.- Rev. Geol. Amér. Central, 44: 9-26.

SCHMIDT, V., 2011b: Factores de amplificación del suelo en función del periodo obtenidos a partir de regresiones para Costa Rica.Rev. Geol. Amér. Central, 44: 27-39.

SPUDICH, P., BOORE, B., FLETCHER, J.B., JOYNER, W.B., LIDNH, A.G. \&
MARGARIS, M., 1999: SEA99: A revised ground motion prediction relations for use in extensional tectonic regimes.- Bull. Seism. Soc. Am. 89(5): 1156-1170.

TAPIA, M., 2006: Desarrollo y aplicación de métodos avanzados para la caracterización de la respuesta sísmica del suelo a escala regional y local.- 355 págs. Univ. Politécnica de Cataluña, Barcelona [Tesis Ph.D].

WARREN, L.M., LANGSTAFF, M.A. \& SILVER, P.G., 2008: Fault plane orientation of intermediate-depth earthquakes in the Middle America Trench.- J. Geophys. Res. 113. B01304. DOI:10.1029/2007JB0050028.

ZHAO, J., ASANO, A., FUKUSHIMA, YA., FUKUSHIMA, YO., IRIKURA, K., OGAWA, H., OHNO, Y., OOCHI, T., SOMERVILLE, P., TAKAHASHI, T., THIO, H.K. \& ZHANG, J., 2006a: Attenuation relations of strong ground motion in Japan using site classification based on predominant period.- BSSA, 96(3): 898-913.

ZHAO, J., ASANO, A., FUKOSHIMA, Y., IRIKURA, K., JOHN, X., OGAWA, H., OHNO, Y., OOUCHI, T., SOMERVILLE, P.G., TAKAHASHI, T. \& ZHANG, J., 2006b: An empirical site-classification method for strong-motion in Japan using $\mathrm{H} / \mathrm{V}$ response spectral ratio.- Bull. Sesism. Soc. Am. 96(3): 914-925. 
